\title{
Classical evolution and quantum generation in generalized gravity theories including string corrections and tachyon: Unified analyses
}

\author{
Jai-chan Hwang ${ }^{(a)}$ and Hyerim $\operatorname{Noh}^{(b)}$ \\ (a) Department of Astronomy and Atmospheric Sciences, Kyungpook National University, Taegu, Korea \\ (b) Korea Astronomy and Space Science Institute, Daejon, Korea \\ E-mails: ${ }^{(a)}$ jchan@knu.ac.kr, ${ }^{(b)}$ hr@kao.re.kr
}

(October 22, 2018)

\begin{abstract}
We present cosmological perturbation theory based on generalized gravity theories including string theory correction terms and a tachyonic complication. The classical evolution as well as the quantum generation processes in these variety of gravity theories are presented in unified forms. These apply both to the scalar- and tensor-type perturbations. Analyses are made based on the curvature variable in two different gauge conditions often used in the literature in Einstein's gravity; these are the curvature variables in the comoving (or uniform-field) gauge and the zero-shear gauge. Applications to generalized slow-roll inflations and its consequent power spectra are derived in unified forms which include wide range of inflationary scenarios based on Einstein's gravity and others.
\end{abstract}

\section{INTRODUCTION}

Cosmological linear perturbation theory [1] has central importance in the current cosmological investigations of the large-scale structure and the cosmic microwave background radiation. A rather successful scenario can be made based on Einstein's gravity with varying use of the diverse (but ordinary) fluids and fields as the energy-momentum content. Relativistic gravity theories more general than Einstein's gravity are ubiquitous in the literature; some are variants of Einstein's gravity while others are more generalized forms with natural correction terms arising in the quantum corrections or in the attempt of unified theories like string/M-theory program. Thus, it would be interesting to formulate corresponding cosmological perturbation analyses in these generalized forms of relativistic gravity theories. It would be naturally more interesting if we could make a unified formulation of handling the perturbations in the context of generalized theories including the Einstein's theory as a case. This is our purpose of the presentation. We consider generalized forms of gravity theories expressed as actions in eqs. $(31,45,74,92,110)$.

In the literature equations in two different gauge conditions are popularly used. In terms of the three-space curvature perturbation $\varphi$ the two gauges are the comoving gauge $v \equiv 0$ (or equivalently in the minimally coupled scalar field, the uniform-field gauge $\delta \phi \equiv 0$ ), and the zero-shear gauge $\chi \equiv 0$ conditions. As each of these two gauge conditions fixes the temporal gauge mode completely the variables are equivalently gauge invariant and correspond to the combinations $\varphi_{v}$ (or $\varphi_{\delta \phi}$ ) and $\varphi_{\chi}$ in eq. (10). In the presence of background curvature we need generalization of $\varphi_{v}$ which we call $\Phi$ to have the unified form, and in the case of the generalized gravity we also need generalization of $\varphi_{\chi}$ which we call $\Psi$ to have the unified form, see eqs. $(22,38,57,84,100)$. In all the gravity theories we are considering we can successfully present the perturbation equations in exactly the same form as in Einstein's gravity. Thus, we can present the consequent classical evolution and quantum generation processes in unified forms. Such unified analyses are practically useful to handle the structure evolution because

"it allows to handle situations when one type of gravity theory switches to another type in the early universe."

In $\S$ II we present our notation, summary of the gauge issue, and the fundamental equations in a gauge-ready form. In $\S$ III we present the closed form equations using the $\Phi$ and $\Psi$ which are generalizations of $\varphi_{v}$ and $\varphi_{\chi}$, respectively, in the cases of the fluid, field, and generalized forms of gravity theories. In $\S \mathrm{IV}$ we present the unified form equations for all the gravity theories considered in this work and present variety of exact and asymptotic solutions available. In $\S \mathrm{V}$ we present the quantum generation process in unified form starting from the action formulation. An exact form of inflation generated power spectrum is derived under an assumption of the background which is quite general so that it includes various inflation scenarios suggested in the literature as cases. Thus, we present the final inflationary spectra in unified forms which can be compared with the cosmic microwave background radiation (CMB) and the large-scale structure observations. Our classical evolution and the quantum generation processes are presented for both the scalar-type and the tensor-type perturbations in unified forms. In §VI we summarize the new discoveries in our presentation and provide a discussion. 


\section{STRATEGY AND BASIC EQUATIONS}

\section{A. Notation}

As the metric we consider the Robertson-Walker spacetime with the scalar- and tensor-type perturbations

$$
d s^{2}=-a^{2}(1+2 \alpha) d \eta^{2}-2 a^{2} \beta_{, \alpha} d \eta d x^{\alpha}+a^{2}\left(g_{\alpha \beta}^{(3)}+2 \varphi g_{\alpha \beta}^{(3)}+2 \gamma_{, \alpha \mid \beta}+2 C_{\alpha \beta}\right) d x^{\alpha} d x^{\beta},
$$

where $a(\eta)$ is the cosmic scale factor and $\eta$ is the conformal time defined as $c d t \equiv a d \eta$. We set $c \equiv 1 \equiv \hbar$. The Greek indices $\alpha, \beta, \gamma, \ldots$ indicate the space, and the Latic indices $a, b, c, \ldots$ indicate the spacetime. The spacetime dependent variables $\alpha, \beta, \gamma$ and $\varphi$ are scalar-type perturbed order variables and $C_{\alpha \beta}$ is a (transverse-tracefree) tensortype variable. Indices of $C_{\alpha \beta}$ are based on $g_{\alpha \beta}^{(3)}$ and a vertical bar indicates a covariant derivative based on $g_{\alpha \beta}^{(3)}$. The three-space metric $g_{\alpha \beta}^{(3)}$ indicates the background comoving three-space part of the Robertson-Walker metric which is spatially homogeneous and isotropic; some of its specific representations are

$$
\begin{aligned}
g_{\alpha \beta}^{(3)} d x^{\alpha} d x^{\beta} & =\frac{d r^{2}}{1-K r^{2}}+r^{2}\left(d \theta^{2}+\sin ^{2} \theta d \phi^{2}\right) \\
& =\frac{1}{\left(1+\frac{K}{4} \bar{r}^{2}\right)^{2}}\left(d x^{2}+d y^{2}+d z^{2}\right) \\
& =d \bar{\chi}^{2}+\left(\frac{1}{\sqrt{K}} \sin (\sqrt{K} \bar{\chi})\right)^{2}\left(d \theta^{2}+\sin ^{2} \theta d \phi^{2}\right)
\end{aligned}
$$

where we have

$$
r \equiv \frac{\bar{r}}{1+\frac{K}{4} \bar{r}^{2}}, \quad \bar{r} \equiv \sqrt{x^{2}+y^{2}+z^{2}}, \quad \bar{\chi} \equiv \int^{r} \frac{d r}{\sqrt{1-K r^{2}}} .
$$

The $K$ is the sign of the three-space curvature.

We ignore the vector-type perturbation (rotation) in this paper, see $\S$ VI for a summary. Our metric convention follows Bardeen's [2]. Our perturbation variables have some kinematic interpretations. The kinematic quantites in the normal frame are [3]:

$$
\theta=3 H-\kappa, \quad \sigma_{\alpha \beta}=\chi_{, \alpha \mid \beta}-\frac{1}{3} g_{\alpha \beta}^{(3)} \Delta \chi+a^{2} \dot{C}_{\alpha \beta}^{(t)}, \quad a_{\alpha}=\alpha_{, \alpha}, \quad R^{(h)}=\frac{1}{a^{2}}[6 K-4(\Delta+3 K) \varphi],
$$

where we introduced

$$
\chi \equiv a(\beta+a \dot{\gamma}), \quad \kappa \equiv \delta K=3 H \alpha-3 \dot{\varphi}-\frac{\Delta}{a^{2}} \chi
$$

An overdot denotes time derivative based on $t, H \equiv \frac{\dot{a}}{a}$, and $\Delta$ is a Laplacian operator based on $g_{\alpha \beta}^{(3)}$. The $\theta, \sigma_{a b}$ and $a_{a}$ are the expansion scalar, shear tensor and the acceleration vector, respectively; in the normal-frame we have the vanishing rotation tensor $\omega_{\alpha \beta}=0$ based on the frame vector [4]. From these we can interprete $\chi, \kappa$ and $\varphi$ as the perturbed shear, the perturbed expansion and the perturbed curvature of the normal-frame vector.

As the energy-momentum tensor we consider an imperfect fluid form including the scalar- and tensor-type perturbations

$$
T_{0}^{0}=-(\bar{\mu}+\delta \mu), \quad T_{\alpha}^{0}=-(\mu+p) v_{, \alpha}, \quad T_{\beta}^{\alpha}=(\bar{p}+\delta p) \delta_{\beta}^{\alpha}+\Pi_{\beta}^{\alpha},
$$

where $\Pi_{\beta}^{\alpha}$ is a tracefree anisotropic stress; $\Pi_{\beta}^{\alpha}$ is based on $g_{\alpha \beta}^{(3)}$. An overbar indicates the background order quantities; we ignore it unless necessary. The cosmological constant $\Lambda$ can be included by adding $T^{(\Lambda) a}=-\frac{\Lambda}{8 \pi G} \delta_{b}^{a}$ to the energymomentum tensor; thus, $\Lambda$ can be included by adding $\mu_{\Lambda}=-p_{\Lambda}=\frac{\Lambda}{8 \pi G}$ to the background fluid quantities $\mu$ and $p$. The entropic perturbation $e$ is defined as

$$
e \equiv \delta p-c_{s}^{2} \delta \mu, \quad c_{s}^{2} \equiv \dot{p} / \dot{\mu} .
$$

We decompose the anisotropic stress as

$$
\Pi_{\alpha \beta} \equiv \frac{1}{a^{2}}\left(\Pi_{, \alpha \mid \beta}-\frac{1}{3} g_{\alpha \beta}^{(3)} \Delta \Pi\right)+\Pi_{\alpha \beta}^{(t)},
$$

where $\Pi_{\alpha \beta}^{(t)}$ is transverse and tracefree. In an ideal fluid we have $e=0$ and $\Pi=0=\Pi_{\alpha \beta}^{(t)}$. 


\section{B. Gauge issue}

Here we summarize the behaviors of our perturbation variables under the gauge transformation and our strategy of how to handle and use such degrees of freedom as advantage. Under the gauge transformation $\hat{x}^{a} \equiv x^{a}+\tilde{\xi}^{a}\left(x^{e}\right)$ we have $[2,3]$

$$
\begin{aligned}
& \hat{\alpha}=\alpha-\dot{\xi}^{t}, \quad \hat{\beta}=\beta-\frac{1}{a} \xi^{t}+a\left(\frac{\xi}{a}\right), \quad \hat{\gamma}=\gamma-\frac{1}{a} \xi, \quad \hat{\varphi}=\varphi-H \xi^{t}, \quad \hat{\chi}=\chi-\xi^{t}, \quad \hat{\kappa}=\kappa+\left(3 \dot{H}+\frac{\Delta}{a^{2}}\right) \xi^{t}, \\
& \delta \hat{\mu}=\delta \mu-\dot{\mu} \xi^{t}, \quad \delta \hat{p}=\delta p-\dot{p} \xi^{t}, \quad \hat{v}=v-\frac{1}{a} \xi^{t}, \quad \hat{\Pi}=\Pi, \quad \delta \hat{\phi}=\delta \phi-\dot{\phi} \xi^{t} ; \quad \hat{C}_{\alpha \beta}=C_{\alpha \beta}, \quad \hat{\Pi}_{\alpha \beta}^{(t)}=\Pi_{\alpha \beta}^{(t)},
\end{aligned}
$$

where we used $\xi^{0} \equiv \frac{1}{a} \xi^{t}$ and $\xi_{\alpha} \equiv \xi_{, \alpha} ; \bar{\phi}$ and $\delta \phi$ are the background and perturbed part of a scalar field $\phi(\mathbf{x}, t)$. Thus, using $\chi$ instead of $\beta$ and $\gamma$ individually, all our perturbation variables are spatially gauge-invariant. However, all our scalar-type perturbation variables depend on the temporal gauge transformation which will be used as advantage in our gauge-ready strategy [3]. Temporal gauge fixing condition, fixing $\xi^{t}$, applies only to the scalar-type perturbation. To the linear-order, we can impose any one of the following temporal gauge conditions to be valid at any spacetime point: the synchronous gauge $(\alpha \equiv 0)$, the comoving gauge $(v \equiv 0)$, the zero-shear gauge $(\chi \equiv 0)$, the uniform-expansion gauge $(\kappa \equiv 0)$, the uniform-curvature gauge $(\varphi \equiv 0)$, the uniform-density gauge $(\delta \mu \equiv 0)$, the uniform-pressure gauge $(\delta p \equiv 0)$, the uniform-field gauge $(\delta \phi \equiv 0)$. Any linear combination of these gauge conditions which can give a constraint on $\xi^{t}$ can be regarded as a suitable temporal gauge condition, thus we have infinite number of temporal gauge conditions available. In the synchronous gauge we sets $\alpha \equiv 0$ in all coordinate systems which leave nonvanishing $\xi^{t}(\mathbf{x})$ with general dependence on spatial coordinate, see eq. (9). Whereas in the other gauge conditions mentioned above we have $\xi^{t}=0$ after imposing any of the temporal gauge condition in all coordinate systems, thus removing the gauge mode completely. Thus, except for the synchronous gauge condition, each of the other temporal gauge fixing conditions completely removes the temporal gauge mode. Later, we will present our fundamental set of scalar-type perturbation equations in a naturally spatially gauge-invariant form but without fixing the temporal gauge conditions. The equations will be arranged so that we can impose any of our fundamental gauge conditions easily depending on the specific problems we encounter; thus we suggestively call our approach a gauge-ready formulation [3].

We introduce several gauge-invariant combinations:

$$
\varphi_{\chi} \equiv \varphi-H \chi, \quad \varphi_{v} \equiv \varphi-a H v, \quad \delta_{v} \equiv \delta-a \frac{\dot{\mu}}{\mu} v, \quad \delta \phi_{\varphi} \equiv \delta \phi-\frac{\dot{\phi}}{H} \varphi \equiv-\frac{\dot{\phi}}{H} \varphi_{\delta \phi}, \quad v_{\chi} \equiv v-\frac{1}{a} \chi \equiv-\frac{1}{a} \chi_{v},
$$

where $\delta \equiv \delta \mu / \mu$. The gauge-invariant combination $\delta \phi_{\varphi}$ is equivalent to $\delta \phi$ in the uniform-curvature gauge which takes $\varphi \equiv 0$ as the gauge condition, etc. Using our notation for the gauge-invariant combinations we can systematically construct and trace various gauge-invariant combinations for a given variable, [3]. As in the last two examples in eq. (10) our notation of gauge-invariant variables allows algebra connecting different expressions of the same gaugeinvariant combinations. Compared with the notation used by Bardeen in 1980 [5], ignoring the harmonic functions, we have:

$$
\Phi_{H} \equiv \varphi_{\chi}, \quad \Phi_{A} \equiv \alpha_{\chi}, \quad \phi_{m} \equiv \varphi_{v}, \quad \epsilon_{m} \equiv \delta_{v}, \quad v_{s}^{(0)} \equiv k v_{\chi}, \quad p \pi_{L}^{(0)} \equiv \delta p, \quad p \pi_{T}^{(0)} \equiv-\frac{\Delta}{a^{2}} \Pi .
$$

Later we will use $\varphi_{v}$ (or $\varphi_{\delta \phi}$ ) and $\varphi_{\chi}$ as the main variables. Considering eqs. (4,6) we may regard the gauge-invariant combinations $\varphi_{v}$ and $\varphi_{\chi}$ as the perturbed three-space curvature $(\varphi)$ in the comoving gauge $(v \equiv 0)$ and the zero-shear gauge $(\chi)$, respectively, based on the normal-frame vector field.

\section{Basic equations}

The background evolution is governed by

$$
H^{2}=\frac{8 \pi G}{3} \mu-\frac{K}{a^{2}}+\frac{\Lambda}{3}, \quad \dot{H}=-4 \pi G(\mu+p)+\frac{K}{a^{2}} .
$$

To the perturbed order, the scalar-type perturbations are described by $[2,3]$ 


$$
\begin{aligned}
& \kappa \equiv 3(-\dot{\varphi}+H \alpha)-\frac{\Delta}{a^{2}} \chi, \\
& \frac{\Delta+3 K}{a^{2}} \varphi+H \kappa=-4 \pi G \delta \mu, \\
& \kappa+\frac{\Delta+3 K}{a^{2}} \chi=12 \pi G a(\mu+p) v, \\
& \dot{\chi}+H \chi-\alpha-\varphi=8 \pi G \Pi, \\
& \dot{\kappa}+2 H \kappa+\left(3 \dot{H}+\frac{\Delta}{a^{2}}\right) \alpha=4 \pi G(\delta \mu+3 \delta p), \\
& \delta \dot{\mu}+3 H(\delta \mu+\delta p)=(\mu+p)\left(\kappa-3 H \alpha+\frac{\Delta}{a} v\right), \\
& \frac{\left[a^{4}(\mu+p) v\right]}{a^{4}(\mu+p)}=\frac{1}{a}\left(\alpha+\frac{\delta p}{\mu+p}+\frac{2}{3} \frac{\Delta+3 K}{a^{2}} \frac{\Pi}{\mu+p}\right) .
\end{aligned}
$$

These follow from Einstein's equations and the energy and momentum conservation equations; eq. (13) is a definition of $\kappa$, eqs. (14-17) follow from $G_{0}^{0}, G_{\alpha}^{0}, G_{\beta}^{\alpha}-\frac{1}{3} \delta_{\beta}^{\alpha} G_{\gamma}^{\gamma}$ and $G_{\alpha}^{\alpha}-G_{0}^{0}$ components of Einstein's equation, respectively, and eqs. $(18,19)$ follow from $T_{0 ; b}^{b}=0$ and $T_{\alpha ; b}^{b}=0$, respectively. These equations are presented without fixing the temporal gauge condition and using the spatially gauge-invariant variables only. Thus, these are presented in a gauge-ready form which allows us to choose the temporal gauge condition depending on the situation as an advantage in handling the problem. This set of equations was first presented by Bardeen in [2]. As we are considering the most general imperfect fluid, the above equations are valid even in the context of generalized gravity theories we are considering: the fluid quantities $\mu, p, \delta \mu, \delta p,(\mu+p) v$, and $\Pi$ can be reinterpreted as the effective fluid quantities, see below eq. (21) and $[6,3]$.

In the case of the gravitational wave we have

$$
\ddot{C}_{\beta}^{\alpha}+3 H \dot{C}_{\beta}^{\alpha}-\frac{\Delta-2 K}{a^{2}} C_{\beta}^{\alpha}=8 \pi G \Pi_{\beta}^{(t) \alpha} .
$$

In eqs. $(12-19,20)$ we presented the complete sets of background and the perturbed equations for a general imperfect fluid. In the case of fluid we include the most general type of imperfect-fluid contributions for both the background and perturbation. In such forms the equations are generally valid for the case of a scalar field and the other generalized gravity theories, by re-interpreting the fluid quantities as the effective fluid quantities. That is, by arranging the gravitational field equation in the following form

$$
G_{b}^{a}=8 \pi G T_{b}^{a}
$$

we re-interprete $T_{b}^{a}$ as the effective energy-momentum tensor [6]. Thus, in those generalized theories we will present only the effective fluid quantities which together with the general equations derived in the fluid in eqs. (12-19) provide complete set of equations.

\section{EQUATIONS IN TWO GAUGES}

\section{A. Fluid}

We introduce the Field-Shepley combination [7]

$$
\Phi \equiv \varphi_{v}-\frac{K / a^{2}}{4 \pi G(\mu+p)} \varphi_{\chi} .
$$

We can derive $[8]$

$$
\begin{aligned}
& \dot{\Phi}=\frac{H}{4 \pi G(\mu+p)} \frac{c_{s}^{2} \Delta}{a^{2}} \varphi_{\chi}-\frac{H}{\mu+p}\left(e+\frac{2}{3} \frac{\Delta}{a^{2}} \Pi\right), \\
& \frac{H}{a}\left(\frac{a}{H} \varphi_{\chi}\right)=\frac{4 \pi G(\mu+p)}{H} \Phi-8 \pi G H \Pi .
\end{aligned}
$$


Equation (23) follows from taking a time derivative of eq. (22) and using eqs. (13-16,19); we also need $v_{\chi}=-\frac{1}{a} \chi_{v}$ in eq. (10). Equation (24) follows from eq. (13) and using eqs. $(15,16)$ with $v_{\chi}=v-\frac{1}{a} \chi=-\frac{1}{a H}\left(\varphi_{v}-\varphi_{\chi}\right)$ which follows from eq. (10). We can combine eqs. $(23,24)$ to make closed form second-order differential equations for both $\Phi$ and $\varphi_{\chi}$

$$
\begin{aligned}
& \frac{H^{2} c_{s}^{2}}{a^{3}(\mu+p)}\left\{\frac{a^{3}(\mu+p)}{H^{2} c_{s}^{2}}\left[\dot{\Phi}+\frac{H}{\mu+p}\left(e+\frac{2}{3} \frac{\Delta}{a^{2}} \Pi\right)\right]\right\}=c_{s}^{2} \frac{\Delta}{a^{2}}\left(\Phi-2 \frac{H^{2}}{\mu+p} \Pi\right), \\
& \frac{\mu+p}{H}\left[\frac{H^{2}}{a(\mu+p)}\left(\frac{a}{H} \varphi_{\chi}\right)+8 \pi G \frac{H^{2}}{\mu+p} \Pi\right]=c_{s}^{2} \frac{\Delta}{a^{2}} \varphi_{\chi}-4 \pi G\left(e+\frac{2}{3} \frac{\Delta}{a^{2}} \Pi\right) .
\end{aligned}
$$

In an ideal fluid we have $e=0=\Pi$. For a pressureless medium we have $c_{s}^{2}=0$, thus instead of eqs. $(23,25)$ we have

$$
\dot{\Phi}=0 .
$$

Equations (23-26) show basic forms of equations valid even in the scalar field and generalized gravity theories to be considered in this paper. Unified forms will be presented in $§ I V$.

In the fluid context it is often convenient to have equation for density perturbation. The most convenient (i.e., similar to the Newtonian) form is available in the comoving gauge, thus equivalently using a gauge-invariant combination $\delta_{v}$, [9]. Using the Poisson's relation

$$
-\frac{\Delta+3 K}{a^{2}} \varphi_{\chi}=4 \pi G \mu \delta_{v},
$$

which follows from eqs. $(14,15)$, eq. (26) gives [9]

$$
\frac{\mu+p}{a^{2} H \mu}\left[\frac{H^{2}}{a(\mu+p)}\left(\frac{a^{3} \mu}{H} \delta_{v}\right)^{\cdot}-2 \frac{H^{2}}{\mu+p}(\Delta+3 K) \Pi\right]^{\cdot}=c_{s}^{2} \frac{\Delta}{a^{2}} \delta_{v}+\frac{\Delta+3 K}{a^{2}} \frac{1}{\mu}\left(e+\frac{2}{3} \frac{\Delta}{a^{2}} \Pi\right) .
$$

For the tensor mode eq. (20) gives

$$
\frac{1}{a^{3}}\left(a^{3} \dot{C}_{\alpha \beta}\right)-\frac{\Delta-2 K}{a^{2}} C_{\alpha \beta}=8 \pi G \Pi_{\alpha \beta}^{(t)} .
$$

The fluid perturbation and the gravitational wave were studied in the context of the synchronous gauge $(\alpha \equiv 0)$ by Lifshitz [1]. The zero-shear gauge $(\chi \equiv 0)$ to handle the gravitational potential $(\varphi)$ and the comoving gauge $(v \equiv 0)$ for the density perturbation $(\delta)$, were first studied by Harrison [10] and Nariai [11], respectively.

\section{B. Field}

We consider an action for a minimally coupled scalar field [12-14]

$$
S=\int d^{4} x \sqrt{-g}\left[\frac{1}{16 \pi G} R-\frac{1}{2} \phi^{, c} \phi_{, c}-V(\phi)\right] .
$$

The gravitational field equation and the equation of motion are

$$
\begin{aligned}
& G_{a b}=8 \pi G\left(\phi_{, a} \phi_{, b}-\frac{1}{2} \phi^{, c} \phi_{, c} g_{a b}-V g_{a b}\right), \\
& \square \phi-V_{, \phi}=0 .
\end{aligned}
$$

Equations (12-19) remain valid with the following background and perturbed order fluid quantities

$$
\begin{aligned}
& \mu \equiv \frac{1}{2} \dot{\phi}^{2}+V, \quad p \equiv \frac{1}{2} \dot{\phi}^{2}-V, \\
& \delta \mu \equiv \dot{\phi} \delta \dot{\phi}-\dot{\phi}^{2} \alpha+V_{, \phi} \delta \phi, \quad \delta p \equiv \dot{\phi} \delta \dot{\phi}-\dot{\phi}^{2} \alpha-V_{, \phi} \delta \phi, \quad v \equiv \frac{1}{a} \frac{\delta \phi}{\dot{\phi}}, \quad \Pi=0=\Pi_{\beta}^{(t) \alpha},
\end{aligned}
$$

where we expanded $\phi(\mathbf{x}, t)=\bar{\phi}(t)+\delta \phi(\mathbf{x}, t)$. Additionally, from eq. (33) we have the background and perturbed equation of motion 


$$
\begin{aligned}
& \ddot{\phi}+3 H \dot{\phi}+V_{, \phi}=0, \\
& \delta \ddot{\phi}+3 H \delta \dot{\phi}-\frac{\Delta}{a^{2}} \delta \phi+V_{, \phi \phi} \delta \phi=\dot{\phi}(\kappa+\dot{\alpha})+(2 \ddot{\phi}+3 H \dot{\phi}) \alpha .
\end{aligned}
$$

As $\delta \phi=0$ implies $v=0$, the uniform-field gauge $(\delta \phi \equiv 0)$ is equivalent to the comoving gauge $(v \equiv 0)$, thus $\varphi_{v}=\varphi_{\delta \phi}$. Thus, eq. (22) becomes

$$
\Phi \equiv \varphi_{\delta \phi}-\frac{K / a^{2}}{4 \pi G \dot{\phi}^{2}} \varphi_{\chi}
$$

We have

$$
\begin{aligned}
& \dot{\Phi}=\frac{H}{4 \pi G \dot{\phi}^{2}} \frac{c_{A}^{2} \Delta}{a^{2}} \varphi_{\chi}, \\
& \frac{H}{a}\left(\frac{a}{H} \varphi_{\chi}\right)=\frac{4 \pi G \dot{\phi}^{2}}{H} \Phi,
\end{aligned}
$$

where

$$
c_{A}^{2} \equiv 1+3\left(1-c_{s}^{2}\right) K \Delta^{-1}, \quad c_{s}^{2} \equiv \frac{\dot{p}}{\dot{\mu}}=-1-\frac{2 \ddot{\phi}}{3 H \dot{\phi}} .
$$

Equation (39) follows from taking a time derivative of eq. (38) and using eqs. (13-16); we also need $\delta \mu_{\delta \phi}=-\dot{\phi}^{2} \alpha_{\delta \phi}$ which follows from eq. (35). Equation (40) follows from eq. (13) and using eqs. $(15,16)$ with $\delta \phi_{\chi}=\delta \phi-\dot{\phi} \chi=$ $\frac{\dot{\phi}}{H}\left(-\varphi_{\delta \phi}+\varphi_{\chi}\right)$ which follows from eq. (10). Equations $(39,40)$ also follow from eqs. $(23,24)$ using the effective fluid quantities in eqs. $(34,35)$ : from eqs. $(34,35)$ we have

$$
e=\left(1-c_{s}^{2}\right) \delta \mu_{\delta \phi}=-\left(1-c_{s}^{2}\right) \frac{\Delta+3 K}{4 \pi G a^{2}} \varphi_{\chi}
$$

where we used eq. (28), and $\delta \mu_{\delta \phi}=\delta \mu_{v}$. Using eq. (42) and $\Pi=0$ in eqs. $(23,24)$ we can derive eqs. (39,40). By combining eqs. $(39,40)$ we have

$$
\begin{aligned}
& \frac{H^{2} c_{A}^{2}}{a^{3} \dot{\phi}^{2}}\left[\frac{a^{3} \dot{\phi}^{2}}{H^{2} c_{A}^{2}} \dot{\Phi}\right]=c_{A}^{2} \frac{\Delta}{a^{2}} \Phi \\
& \frac{\dot{\phi}^{2}}{H}\left[\frac{H^{2}}{a \dot{\phi}^{2}}\left(\frac{a}{H} \varphi_{\chi}\right)\right]^{\cdot}=c_{A}^{2} \frac{\Delta}{a^{2}} \varphi_{\chi}
\end{aligned}
$$

which can be compared with the ideal fluid (thus sets $e \equiv 0 \equiv \Pi$ ) equations in eqs. (25,26). Thus, we have an interesting conclusion:

"Compared with an ideal fluid, the minimally coupled scalar field effectively has $c_{s}^{2}$ replaced by $c_{A}^{2}$ which becomes 1 for $K=0 . "$

The $c_{A}$ has the role of wave speed of the perturbed field and the simultaneously excited metric; interpretation of $c_{A}$ as the wave speed is properly valid only for $K=0$. Thus, for $K=0$ the wave propagation speed becomes 1 . In the minimally coupled scalar field $c_{s}$ cannot be interpreted as the wave propagation speed. This is because the scalar field has a non-vanishing entropic perturbation as in eq. (42), thus cannot be interpreted as an ideal fluid.

For the tensor mode, eq. (30) remains valid in the field situation with $\Pi_{\alpha \beta}^{(t)}=0$. That is, the presence of minimally coupled scalar field (or fields) does not directly affect the equation of tensor-type perturbation.

\section{Generalized $f(\phi, R)$ gravity}

We consider an action $[6,3,15-17]$

$$
S=\int d^{4} x \sqrt{-g}\left[\frac{1}{2} f(\phi, R)-\frac{1}{2} \omega(\phi) \phi^{, c} \phi_{, c}-V(\phi)+L_{(m)}+L_{(c)}\right],
$$


where $L_{(c)}$ represents additional correction terms to be considered in $\S I I I F$ and III G. This action without $L_{(c)}$ includes the following gravity theories as subset: $f(R)$ gravity which includes $R^{2}$ gravity, the scalar-tensor theory which includes the Jordan-Brans-Dicke theory, the non-minimally coupled scalar field, the induced gravity, the lowenergy effective action of string theory, etc, see [17]. Although this generalized action by itself does not have much physical meaning, there are some advantages by analysing perturbations in this general context: for example, our results will be valid considering transitions from one type of gravity theory to the other. The gravitational field equation and the equation of motion are

$$
\begin{aligned}
& G_{a b}=\frac{1}{F}\left[T_{a b}^{(m)}+\omega\left(\phi_{, a} \phi_{, b}-\frac{1}{2} \phi^{, c} \phi_{, c} g_{a b}\right)+\frac{1}{2}(f-R F-2 V) g_{a b}+F_{, a ; b}-\square F g_{a b}+T_{a b}^{(c)}\right], \\
& \square \phi+\frac{1}{2 \omega}\left(\omega_{, \phi} \phi^{, c} \phi_{, c}+f_{, \phi}-2 V_{, \phi}\right)=\frac{1}{2 \omega} T^{(c)},
\end{aligned}
$$

where $F \equiv \frac{\partial f}{\partial R}$. We have

$$
\delta\left(\sqrt{-g} L_{(m)}\right) \equiv \frac{1}{2} \sqrt{-g} T_{(m)}^{a b} \delta g_{a b}, \quad \delta\left(\sqrt{-g} L_{(c)}\right) \equiv \frac{1}{2} \sqrt{-g} T_{(c)}^{a b} \delta g_{a b} .
$$

Equations (12-20) remain valid with the following effective fluid quantities

$$
\begin{aligned}
8 \pi G \mu= & \frac{1}{F}\left(\frac{1}{2} \omega \dot{\phi}^{2}+\frac{R F-f+2 V}{2}-3 H \dot{F}-T_{0}^{(c) 0} 0\right) \\
8 \pi G p= & \frac{1}{F}\left(\frac{1}{2} \omega \dot{\phi}^{2}-\frac{R F-f+2 V}{2}+\ddot{F}+2 H \dot{F}+\frac{1}{3} T_{\alpha}^{(c) \alpha}\right) \\
8 \pi G \delta \mu= & \frac{1}{F}\left[\omega \dot{\phi} \delta \dot{\phi}+\frac{1}{2}\left(\omega_{, \phi} \dot{\phi}^{2}-f_{, \phi}+2 V_{, \phi}\right) \delta \phi-3 H \delta \dot{F}+\left(3 \dot{H}+3 H^{2}+\frac{\Delta}{a^{2}}\right) \delta F\right. \\
& \left.+\left(3 H \dot{F}-\omega \dot{\phi}^{2}\right) \alpha+\dot{F} \kappa-\left(\delta T_{0}^{(c) 0}-\frac{\delta F}{F} T_{0}^{(c) 0}\right)\right] \\
8 \pi G \delta p= & \frac{1}{F}\left[\omega \dot{\phi} \delta \dot{\phi}+\frac{1}{2}\left(\omega_{, \phi} \dot{\phi}^{2}+f_{, \phi}-2 V_{, \phi}\right) \delta \phi+\delta \ddot{F}+2 H \delta \dot{F}+\left(-\dot{H}-3 H^{2}-\frac{2}{3} \frac{\Delta+3 K}{a^{2}}\right) \delta F\right. \\
& \left.-\dot{F} \dot{\alpha}-\left(\omega \dot{\phi}^{2}+2 \ddot{F}+2 H \dot{F}\right) \alpha-\frac{2}{3} \dot{F} \kappa+\frac{1}{3}\left(\delta T_{\alpha}^{(c) \alpha}-\frac{\delta F}{F} T_{\alpha}^{(c) \alpha}\right)\right] \\
8 \pi G T_{\alpha}^{0}= & \frac{1}{F}\left[\frac{1}{a}(-\omega \dot{\phi} \delta \phi-\delta \dot{F}+H \delta F+\dot{F} \alpha)+T_{, \alpha}^{(c) 0} \underset{\alpha}{\alpha}\right], \\
8 \pi G \Pi_{\beta}^{\alpha}= & \frac{1}{F}\left[\frac{1}{a^{2}}\left(\nabla^{\alpha} \nabla_{\beta}-\frac{1}{3} \delta_{\beta}^{\alpha} \Delta\right)(\delta F-\dot{F} \chi)-\dot{F} \dot{C}_{\beta}^{\alpha}+\delta T_{\beta}^{(c) \alpha}-\frac{1}{3} \delta_{\beta}^{\alpha} \delta T_{\gamma}^{(c) \gamma}\right]
\end{aligned}
$$

where we have

$$
\begin{aligned}
& R=6\left(2 H^{2}+\dot{H}+\frac{K}{a^{2}}\right) \\
& \delta R=2\left[-\dot{\kappa}-4 H \kappa-\left(\frac{\Delta}{a^{2}}+3 \dot{H}\right) \alpha-2 \frac{\Delta+3 K}{a^{2}} \varphi\right]=\delta \mu-3 \delta p .
\end{aligned}
$$

We have set $T_{a b}^{(m)}=0$. The scalar- and tensor-type fluid quantities can be read from eqs. $(6,8)$ as

$$
\begin{aligned}
& (\mu+p) v=-\Delta^{-1} \nabla^{\alpha} T_{\alpha}^{0}, \\
& \Pi=\frac{3}{2} a^{2} \Delta^{-1}(\Delta+3 K)^{-1}\left(\Pi_{\beta}^{\alpha \mid \beta}\right), \quad \Pi_{\beta}^{(t) \alpha}=\Pi_{\beta}^{\alpha}-\frac{1}{a^{2}}\left(\nabla^{\alpha} \nabla_{\beta}-\frac{1}{3} \delta_{\beta}^{\alpha} \Delta\right) \Pi, \quad \Pi_{\beta}^{\alpha}=T_{\beta}^{\alpha}-\frac{1}{3} \delta_{\beta}^{\alpha} T_{\gamma}^{\gamma} .
\end{aligned}
$$

The comoving gauge $(v \equiv 0)$ differs from the uniform-field gauge $(\delta \phi \equiv 0)$, thus $\varphi_{\delta \phi} \neq \varphi_{v}$. The equation of motion gives 


$$
\begin{aligned}
& \ddot{\phi}+3 H \dot{\phi}+\frac{1}{2 \omega}\left(\omega_{, \phi} \dot{\phi}^{2}-f_{, \phi}+2 V_{, \phi}\right)=-\frac{1}{2 \omega} T^{(c)} . \\
& \delta \ddot{\phi}+\left(3 H+\frac{\omega_{, \phi}}{\omega} \dot{\phi}\right) \delta \dot{\phi}+\left[-\frac{\Delta}{a^{2}}+\left(\frac{\omega_{, \phi}}{\omega}\right)_{, \phi} \frac{\dot{\phi}^{2}}{2}+\left(\frac{-f_{, \phi}+2 V_{, \phi}}{2 \omega}\right)_{, \phi}\right] \delta \phi \\
& \quad=\dot{\phi} \dot{\alpha}+\left(2 \ddot{\phi}+3 H \dot{\phi}+\frac{\omega_{, \phi}}{\omega} \dot{\phi}^{2}\right) \alpha+\dot{\phi} \kappa+\frac{1}{2 \omega} F_{, \phi} \delta R-\frac{1}{2 \omega}\left(\delta T^{(c)}-\frac{\omega_{, \phi}}{\omega} \delta \phi T^{(c)}\right) .
\end{aligned}
$$

We have additionally located $T_{a b}^{(c)}$ and $T^{(c)}$ terms for later consideration of the string theory correction terms in $\S$ III F and III G. In this subsection we ignore these correction terms. From eqs. $(50,53,6)$ we have

$$
\begin{aligned}
& 8 \pi G(\mu+p) v=\frac{1}{a F}(\omega \dot{\phi} \delta \phi+\delta \dot{F}-H \delta F-\dot{F} \alpha), \\
& 8 \pi G \Pi=\frac{1}{F}(\delta F-\dot{F} \chi), \quad 8 \pi G \Pi_{\beta}^{(t) \alpha}=-\frac{\dot{F}}{F} C_{\beta}^{\alpha} .
\end{aligned}
$$

Instead of eq. (38) we introduce a more generalized form

$$
\Phi \equiv \varphi_{\delta \phi}-2 \frac{K}{a^{2}} \frac{F}{\omega \dot{\phi}^{2}+\frac{3 \dot{F}^{2}}{2 F}} \Psi, \quad \Psi \equiv \varphi_{\chi}+\frac{\delta F_{\chi}}{2 F}
$$

From eqs. (13-15) and eqs. (13,15,16), respectively, we can derive

$$
\begin{aligned}
& \dot{\Phi}=\frac{2 H F+\dot{F}}{\omega \dot{\phi}^{2}+\frac{3 \dot{F}^{2}}{2 F}} \frac{c_{A}^{2} \Delta}{a^{2}} \Psi, \\
& \frac{H+\frac{\dot{F}}{2 F}}{a F}\left(\frac{a F}{H+\frac{\dot{F}}{2 F}} \Psi\right)=\frac{\omega \dot{\phi}^{2}+\frac{3 \dot{F}^{2}}{2 F}}{2 H F+\dot{F}} \Phi,
\end{aligned}
$$

where

$$
c_{A}^{2} \equiv 1+\left(6+\frac{2 \frac{\ddot{\phi}}{\dot{\phi}}-3 \frac{\dot{F}}{F}+\frac{\dot{E}}{E}}{H+\frac{\dot{F}}{2 F}}\right) K \Delta^{-1}, \quad E \equiv F\left(\omega+\frac{3 \dot{F}^{2}}{2 F \dot{\phi}^{2}}\right) .
$$

In order to derive eqs. $(58,59)$ we follow the same algebraic procedure as needed in the scalar field. By combining eqs. $(58,59)$ we have

$$
\begin{aligned}
& \frac{\left(H+\frac{\dot{F}}{2 F}\right)^{2} c_{A}^{2}}{a^{3}\left(\omega \dot{\phi}^{2}+\frac{3 \dot{F}^{2}}{2 F}\right)}\left[\frac{a^{3}\left(\omega \dot{\phi}^{2}+\frac{3 \dot{F}^{2}}{2 F}\right)}{\left(H+\frac{\dot{F}}{2 F}\right)^{2} c_{A}^{2}} \dot{\Phi}\right]=c_{A}^{2} \frac{\Delta}{a^{2}} \Phi, \\
& \frac{\omega \dot{\phi}^{2}+\frac{3 \dot{F}^{2}}{2 F}}{H F+\frac{1}{2} \dot{F}}\left[\frac{\left(H+\frac{\dot{F}}{2 F}\right)^{2}}{a\left(\omega \dot{\phi}^{2}+\frac{3 \dot{F}^{2}}{2 F}\right)}\left(\frac{a F}{H+\frac{\dot{F}}{2 F}} \Psi\right) \cdot\right]=c_{A}^{2} \frac{\Delta}{a^{2}} \Psi .
\end{aligned}
$$

We notice that $c_{A}$ can be interpreted as a wave speed of the perturbed field as well as the simultaneously excited metric; for $K=0$ we have

$$
c_{A}=1,
$$

and only in this case we can properly interprete $c_{A}$ as the wave speed; $c_{A}^{2}$ clearly differs from $c_{s}^{2} \equiv \dot{p} / \dot{\mu}$.

This unified result is valid for the gravities in the forms either (i) $F=F(\phi)$ or (ii) $F=F(R)$. In the case of $f=f(R)$ without the field the results in the above remain valid with the following prescription: we remove $\phi(\omega$ as well) thus we have $E=\frac{3}{2} \dot{F}^{2}$, and set $\varphi_{\delta \phi}=\varphi_{\delta F}$. In the general case with $F=F(\phi, R)$ the situation corresponds to the two component medium, see $\S I I I D$.

Using 


$$
\delta F_{\chi}=\frac{\dot{F}}{H}\left(\varphi_{\chi}-\varphi_{\delta \phi}\right)
$$

we have

$$
\varphi_{\chi}=\frac{H \Psi+\frac{\dot{F}}{2 F} \varphi_{\delta \phi}}{H+\frac{\dot{F}}{2 F}}
$$

As we are considering the linear theory, if the solution of one variable is known in any one gauge, solutions of all the other variables in the same gauge as well as in any other gauge can be derived easily through linear algebra; our gauge-ready form equations in eqs. (13-19) and our convention of the gauge invariant variables in eq. (10) are useful to derive the remaining variables systematically.

For the tensor mode we have

$$
\frac{1}{a^{3} F}\left(a^{3} F \dot{C}_{\alpha \beta}\right) \cdot \frac{\Delta-2 K}{a^{2}} C_{\alpha \beta}=0
$$

This equation is valid for general algebraic function of $f(\phi, R)$. In the context of fluid formulation in eq. (30), the $F$ term in eq. (66) comes from the nonvanishing effective anisotropic stress of our generalized gravity theory in eq. (56).

\section{Conformal transformation}

Although direct derivation of eqs. $(58,59)$ is not complicated there is another simple way. The gravity theory in eq. (45) can be transformed to Einstein's gravity through a conformal rescaling of the metric and rescaling of the field. The end result is Einstein's gravity with only complications appearing in the modified form of the field potential. Thus, using such transformation properties we can derive the equations in generalized gravity from equations in $\S$ III B.

We have studied the conformal transformation properties in $[6,18]$, and in most general form in the Appendix A of [15]. Under the conformal transformation

$$
\hat{g}_{a b}=\Omega^{2} g_{a b}, \quad \Omega \equiv \sqrt{8 \pi G F} \equiv e^{\psi / \sqrt{6}}
$$

with

$$
d \hat{\phi} \equiv \sqrt{\frac{1}{8 \pi G}\left(\frac{\omega}{F} d \phi^{2}+d \psi^{2}\right)}
$$

eq. (45) transforms to Einstein's gravity with a modified potential

$$
\hat{V}=\frac{1}{(16 \pi G F)^{2}}(2 V-f+R F) .
$$

The form of $d \hat{\phi}$ implies that, in order to have Einstein's gravity with one minimally coupled scalar field we need certain condition on the form of $f(\phi, R)$. We need either $f=F(\phi) R$ or a pure $f(R)$ gravity without the field; if we have $f$ a nonlinear function of $R$ with a field involved, we will have Einstein's gravity with two scalar fields.

Under such a transformation, with $\Omega \equiv \bar{\Omega}(1+\delta \Omega)$, we have, to the background order

$$
\hat{a}=a \Omega, \quad d \hat{t}=\Omega d t, \quad \hat{H}=\frac{1}{\Omega}\left(H+\frac{\dot{\Omega}}{\Omega}\right), \quad \dot{\hat{\phi}}=\sqrt{\frac{1}{8 \pi G}\left(\frac{\omega}{F} \dot{\phi}^{2}+\frac{3 \dot{F}^{2}}{2 F^{2}}\right)},
$$

and to the perturbed order

$$
\hat{\varphi}=\varphi+\delta \Omega, \quad \hat{\alpha}=\alpha+\delta \Omega, \quad \hat{\chi}=\Omega \chi, \quad \frac{\delta \hat{\phi}}{\dot{\hat{\phi}}}=\frac{\delta \phi}{\dot{\phi}}=\frac{\delta F}{\dot{F}}
$$

where

$$
\Omega=\sqrt{8 \pi G F}, \quad \delta \Omega=\frac{\delta F}{2 F} .
$$


The following variables are invariant under the conformal transformation

$$
d \eta, \quad \Delta, \quad k^{2}, \quad \varphi_{\delta \phi}=-\frac{H}{\dot{\phi}} \delta \phi_{\varphi}, \quad C_{\alpha \beta},
$$

where $k$ is the comoving wave number with $\Delta=-k^{2}$ in the Fourier space. It would be a trivial exercise to derive eqs. $(58,59)$ from eqs. $(39,40)$ using eqs. $(70-73)$. We can also show that under the conformal transformation we have $\hat{\Phi}=\Phi$ and $\hat{\varphi}_{\hat{\chi}}=\Psi$. Notice that $\varphi_{\delta \phi}$ and $C_{\alpha \beta}$ are invariant under the conformal transformation. From this result we can draw an important conclusion that

"the power spectra (both amplitudes and spectral slopes) based on $\varphi_{\delta \phi}$ and $C_{\alpha \beta}$ derived in the context of any given frame is valid in any other frame."

However, for the two other types of gravity theories to be considered in the following subsections we have no such transformation property available. We have to derive the equations directly from the gravity theories and equations of motion. The same algebraic procedures needed for the scalar field also apply to these gravity theories in a rather exact manner.

\section{E. Tachyonic generalization}

We consider an action [19]

$$
S=\int d^{4} x \sqrt{-g}\left[\frac{1}{2} f(R, \phi, X)+L_{(m)}+L_{(c)}\right]
$$

where $X \equiv \frac{1}{2} \phi^{, c} \phi_{, c}$, and $L_{(c)}$ includes additional correction terms to be considered in $\S I I I F$ and III G. This action includes the generalized $f(\phi, R)$ gravity in eq. (45) as a case. The gravitational field equation and the equation of motion are

$$
\begin{aligned}
& G_{a b}=\frac{1}{F}\left[T_{a b}^{(m)}+\frac{1}{2}(f-R F) g_{a b}+F_{, a ; b}-\square F g_{a b}-\frac{1}{2} f_{, X} \phi_{, a} \phi_{, b}+T_{a b}^{(c)}\right], \\
& \left(f_{, X} \phi^{, c}\right)_{; c}=f_{, \phi}-T^{(c)} .
\end{aligned}
$$

Equations (12-20) remain valid with the following effective fluid quantities

$$
\begin{aligned}
8 \pi G \mu= & \frac{1}{F}\left(f_{, X} X+\frac{F R-f}{2}-3 H \dot{F}-T_{0}^{(c) 0} 0\right) \\
8 \pi G p= & \frac{1}{F}\left(-\frac{F R-f}{2}+\ddot{F}+2 H \dot{F}+\frac{1}{3} T_{\alpha}^{(c) \alpha}\right) \\
8 \pi G \delta \mu= & \frac{1}{F}\left[-\frac{1}{2}\left(f_{, \phi} \delta \phi+f_{, X} \delta X\right)-\frac{1}{2} \dot{\phi}^{2}\left(F_{, X} \delta R+f_{, X \phi} \delta \phi+f_{, X X} \delta X\right)\right. \\
& \left.-f_{, X} \dot{\phi} \delta \dot{\phi}-3 H \delta \dot{F}+\left(3 \dot{H}+3 H^{2}+\frac{\Delta}{a^{2}}\right) \delta F+\dot{F} \kappa+\left(3 H \dot{F}+f_{, X} \dot{\phi}^{2}\right) \alpha-\left(\delta T_{0}^{(c) 0}-\frac{\delta F}{F} T_{0}^{(c) 0}\right)\right], \\
8 \pi G \delta p= & \frac{1}{F}\left[\frac{1}{2}\left(f_{, \phi} \delta \phi+f_{, X} \delta X\right)+\delta \ddot{F}+2 H \delta \dot{F}+\left(-\dot{H}-3 H^{2}-\frac{2}{3} \frac{\Delta+3 K}{a^{2}}\right) \delta F\right. \\
& \left.-\frac{2}{3} \dot{F} \kappa-\dot{F} \dot{\alpha}-2(\ddot{F}+H \dot{F}) \alpha+\frac{1}{3}\left(\delta T_{\alpha}^{(c) \alpha}-\frac{\delta F}{F} T_{\alpha}^{(c) \alpha}\right)\right] \\
8 \pi G T_{\alpha}^{0}= & \frac{1}{F}\left[\frac{1}{a}\left(\frac{1}{2} f_{, X} \dot{\phi} \delta \phi-\delta \dot{F}+H \delta F+\dot{F} \alpha\right)_{, \alpha}+T_{\alpha}^{(c) 0}\right], \\
8 \pi G \Pi_{\beta}^{\alpha}= & \frac{1}{F}\left[\frac{1}{a^{2}}\left(\nabla^{\alpha} \nabla_{\beta}-\frac{1}{3} \delta_{\beta}^{\alpha} \Delta\right)(\delta F-\dot{F} \chi)_{\beta} \dot{F}_{\beta}^{\alpha}+\delta T_{\beta}^{(c) \alpha}-\frac{1}{3} \delta_{\beta}^{\alpha} \delta T_{\gamma}^{(c) \gamma}\right]
\end{aligned}
$$


where we have

$$
X=-\frac{1}{2} \dot{\phi}^{2}, \quad \delta X=-\dot{\phi} \delta \dot{\phi}+\dot{\phi}^{2} \alpha,
$$

and $R$ and $\delta R$ are given in eqs. (51,52). The equation of motion gives

$$
\begin{aligned}
& \frac{1}{a^{3}}\left(a^{3} \dot{\phi} f_{, X}\right)+f_{, \phi}=T^{(c)}, \\
& f_{, X}\left[\delta \ddot{\phi}+\left(3 H+\frac{\dot{f}, X}{f_{, X}}\right) \delta \dot{\phi}-\frac{\Delta}{a^{2}} \delta \phi+\dot{\phi}\left(3 \dot{\varphi}-\dot{\alpha}+\frac{\Delta}{a^{2}} \chi\right)\right]+2 f_{, \phi} \alpha+\frac{1}{a^{3}}\left(a^{3} \dot{\phi} \delta f_{, X}\right)+\delta f_{, \phi}=\delta T^{(c)},
\end{aligned}
$$

where

$$
\delta f=f_{, \phi} \delta \phi+f_{, X} \delta X+f_{, R} \delta R
$$

We have located $T_{a b}^{(c)}$ and $T^{(c)}$ terms for later consideration of the string theory correction terms. In the following we ignore the correction terms. From eqs. $(50,53,6)$ we have

$$
\begin{aligned}
& 8 \pi G(\mu+p) v=-\frac{1}{a F}\left(\frac{1}{2} f_{, X} \dot{\phi} \delta \phi-\delta \dot{F}+H \delta F+\dot{F} \alpha\right), \\
& 8 \pi G \Pi=\frac{1}{F}(\delta F-\dot{F} \chi), \quad 8 \pi G \Pi_{\beta}^{(t) \alpha}=-\frac{\dot{F}}{F} C_{\beta}^{\alpha} .
\end{aligned}
$$

We assume $F=F(\phi)$. From eq. (83) we notice that the uniform-field gauge differs from the comoving gauge. Instead of eq. (38) we introduce a more generalized form

$$
\Phi \equiv \varphi_{\delta \phi}-\frac{K}{a^{2}} \frac{2 F}{X f_{, X}+\frac{3 \dot{F}^{2}}{2 F}} \Psi
$$

where $\Psi$ is the same as in eq. (57). From eqs. (13-15) and eqs. $(13,15,16)$, respectively, we can derive

$$
\begin{aligned}
& \dot{\Phi}=\frac{2 H F+\dot{F}}{X f_{, X}+\frac{3 \dot{F}^{2}}{2 F}} \frac{c_{A}^{2} \Delta}{a^{2}} \Psi, \\
& \frac{H+\frac{\dot{F}}{2 F}}{a F}\left(\frac{a F}{H+\frac{\dot{F}}{2 F}} \Psi\right)=\frac{X f_{, X}+\frac{3 \dot{F}^{2}}{2 F}}{2 H F+\dot{F}} \Phi,
\end{aligned}
$$

where

$$
\begin{aligned}
c_{A}^{2} & \equiv \frac{X f_{, X}+\frac{3 \dot{F}^{2}}{2 F}}{X f_{, X}+2 X^{2} f_{, X X}+\frac{3 \dot{F}^{2}}{2 F}}\left\{1+\left[3+\frac{X\left(f_{, X}+2 X f_{, X X}\right)+\frac{3 \dot{F}^{2}}{2 F}}{X f_{, X}+\frac{3 \dot{F}^{2}}{2 F}}\left(3+\frac{\frac{\dot{X}}{X}-3 \frac{\dot{F}}{F}+\frac{\dot{E}}{E}}{H+\frac{\dot{F}}{2 F}}\right)\right] K \Delta^{-1}\right\}, \\
E & \equiv-\frac{F}{2 X}\left(X f_{, X}+\frac{3 \dot{F}^{2}}{2 F}\right) .
\end{aligned}
$$

Equation (65) remains valid. By combining eqs. $(85,86)$ we have

$$
\begin{aligned}
& \frac{\left(H+\frac{\dot{F}}{2 F}\right)^{2} c_{A}^{2}}{a^{3}\left(X f_{, X}+\frac{3 \dot{F}^{2}}{2 F}\right)}\left[\frac{a^{3}\left(X f_{, X}+\frac{3 \dot{F}^{2}}{2 F}\right)}{\left(H+\frac{\dot{F}}{2 F}\right)^{2} c_{A}^{2}} \dot{\Phi}\right]=c_{A}^{2} \frac{\Delta}{a^{2}} \Phi, \\
& \frac{X f_{, X}+\frac{3 \dot{F}^{2}}{2 F}}{H F+\frac{1}{2} \dot{F}}\left[\frac{\left(H+\frac{\dot{F}}{2 F}\right)^{2}}{a\left(X f_{, X}+\frac{3 \dot{F}^{2}}{2 F}\right)}\left(\frac{a F}{H+\frac{\dot{F}}{2 F}} \Psi\right)\right)^{\cdot}=c_{A}^{2} \frac{\Delta}{a^{2}} \Psi .
\end{aligned}
$$

$c_{A}^{2}$ differs clearly from $c_{s}^{2} \equiv \dot{p} / \dot{\mu}$. Contrary to the minimally coupled scalar field and the generalized $f(\phi, R)$ gravity theory the wave speed is non-trivial even for $K=0$. For $K=0$ we have

$$
c_{A}^{2}=\frac{X f_{, X}+\frac{3 \dot{F}^{2}}{2 F}}{X f_{, X}+2 X^{2} f_{, X X}+\frac{3 \dot{F}^{2}}{2 F}} .
$$

For the tensor mode, eq. (66) remains valid exactly. 


\section{F. String corrections}

We consider an action in eq. (45) with the following additional corrections in the action [20,21]

$$
L_{(c)}=-\frac{1}{2} \xi(\phi)\left[c_{1} R_{G B}^{2}+c_{2} G^{a b} \phi_{, a} \phi_{, b}+c_{3} \square \phi \phi^{, c} \phi_{, c}+c_{4}\left(\phi^{, c} \phi_{, c}\right)^{2}\right],
$$

where $R_{G B}^{2} \equiv R^{a b c d} R_{a b c d}-4 R^{a b} R_{a b}+R^{2}$. Corrections to the gravitational field equation and the equation of motion are $^{1}$

$$
\begin{aligned}
T_{a b}^{(c)}= & -c_{1}\left[\left(\frac{1}{2} R_{G B}^{2} g_{a b}+4 R_{a c} R_{b}^{c}+4 R^{c d} R_{a c b d}-2 R_{a}{ }^{c d e} R_{b c d e}-2 R R_{a b}\right) \xi\right. \\
& \left.-4\left(\xi ; c d R_{a c b d}-\square \xi R_{a b}+2 \xi_{; c(b} R_{a)}^{c}-\frac{1}{2} \xi_{, a ; b} R\right)+2\left(2 \xi_{; c d} R^{c d}-\square \xi R\right) g_{a b}\right] \\
& -c_{2}\left\{\xi\left(\frac{1}{2} R_{a b} \phi^{, c} \phi_{, c}+\frac{1}{2} R \phi_{, a} \phi_{, b}-2 R_{(a}^{c} \phi_{, b)} \phi_{, c}\right)-\frac{1}{2} \square\left(\xi \phi_{, a} \phi_{, b}\right)-\frac{1}{2}\left(\xi \phi^{, c} \phi_{, c}\right)_{, a ; b}+\left(\xi \phi^{, c} \phi_{,(a}\right)_{; b) c}\right. \\
& \left.+\frac{1}{2}\left[\xi G^{c d} \phi_{, c} \phi_{, d}-\left(\xi \phi^{, c} \phi^{, d}\right)_{; c d}+\square\left(\xi \phi^{, c} \phi_{, c}\right)\right] g_{a b}\right\} \\
& -c_{3}\left[\left(\xi \phi^{, c} \phi_{, c}\right)_{,(a} \phi_{, b)}-\xi \square \phi \phi_{, a} \phi_{, b}-\frac{1}{2}\left(\xi \phi^{, c} \phi_{, c}\right)_{, d} \phi^{, d} g_{a b}\right]-c_{4} \xi \phi^{, c} \phi_{, c}\left(-2 \phi_{, a} \phi_{, b}+\frac{1}{2} \phi^{, d} \phi_{, d} g_{a b}\right), \\
T^{(c)}= & c_{1} \xi_{, \phi} R_{G B}^{2}-c_{2} G^{a b}\left(\xi_{, \phi} \phi \phi_{, a} \phi_{, b}+2 \xi \phi_{, a ; b}\right)+c_{3}\left[\xi_{, \phi} \square \phi \phi^{, a} \phi_{, a}+\square\left(\xi \phi^{, a} \phi_{, a}\right)-2\left(\xi \square \phi \phi^{, a}\right)_{; a}\right] \\
& +c_{4}\left[\xi \xi_{, \phi}\left(\phi^{, a} \phi_{, a}\right)^{2}-4\left(\xi \phi^{, a} \phi^{, b} \phi_{, b}\right)_{; a}\right] .
\end{aligned}
$$

The first line in RHS of eq. (93) vanishes because we have $\delta \int \sqrt{-g} R_{G B}^{2} d^{4} x=0$.

Equations $(12,19)$ remain valid with the same effective fluid quantities in eqs. $(49,50)$ and the following correction terms. To the background order we have

$$
\begin{aligned}
T_{0}^{(c) 0}= & -12 c_{1} H\left(H^{2}+\frac{K}{a^{2}}\right) \dot{\xi}+\frac{3}{2} c_{2}\left(3 H^{2}+\frac{K}{a^{2}}\right) \xi \dot{\phi}^{2}-\frac{1}{2} c_{3}(\dot{\xi}-6 H \xi) \dot{\phi}^{3}+\frac{3}{2} c_{4} \xi \dot{\phi}^{4}, \\
T_{\alpha}^{(c) \alpha}= & -12 c_{1}\left[\left(H^{2}+\frac{K}{a^{2}}\right) \ddot{\xi}+2 H\left(\dot{H}+H^{2}\right) \dot{\xi}\right]+\frac{3}{2} c_{2} \dot{\phi}\left[\left(2 \dot{H}+3 H^{2}-\frac{K}{a^{2}}\right) \xi \dot{\phi}+4 H \xi \ddot{\phi}+2 H \dot{\xi} \dot{\phi}\right] \\
& +\frac{3}{2} c_{3} \dot{\phi}^{2}(2 \xi \ddot{\phi}+\dot{\xi} \dot{\phi})-\frac{3}{2} c_{4} \xi \dot{\phi}^{4}, \\
T^{(c)}= & 24 c_{1}\left(\dot{H}+H^{2}\right)\left(H^{2}+\frac{K}{a^{2}}\right) \xi_{, \phi}+3 c_{2}\left[-\left(H^{2}+\frac{K}{a^{2}}\right)(\dot{\xi} \dot{\phi}+2 \xi \ddot{\phi})-2 H\left(2 \dot{H}+3 H^{2}+\frac{K}{a^{2}}\right) \xi \dot{\phi}\right] \\
& +c_{3} \dot{\phi}\left[\ddot{\xi} \dot{\phi}+3 \dot{\xi} \ddot{\phi}-6 \xi\left(\dot{H} \dot{\phi}+2 H \ddot{\phi}+3 H^{2} \dot{\phi}\right)\right]+c_{4} \dot{\phi}^{2}(-3 \dot{\xi} \dot{\phi}-12 \xi \ddot{\phi}-12 H \xi \dot{\phi}) .
\end{aligned}
$$

To the perturbed order, assuming $K=0$, we have ${ }^{2}$

$$
\begin{aligned}
\delta T_{0}^{(c) 0}= & -4 c_{1} H\left[3 H^{2} \delta \dot{\xi}-3 H \dot{\xi}(\kappa+H \alpha)-\frac{\Delta}{a^{2}}(H \delta \xi+2 \dot{\xi} \varphi)\right] \\
& +c_{2} \dot{\phi}\left[\frac{9}{2} H^{2} \dot{\phi} \delta \xi+9 H^{2} \xi \delta \dot{\phi}-2 H \xi \frac{\Delta}{a^{2}} \delta \phi-\xi \dot{\phi}\left(3 H \kappa+9 H^{2} \alpha+\frac{\Delta}{a^{2}} \varphi\right)\right] \\
& -\frac{1}{2} c_{3} \dot{\phi}^{2}\left[3(\dot{\xi}-6 H \xi) \delta \dot{\phi}+2 \xi \frac{\Delta}{a^{2}} \delta \phi+\dot{\phi}(\delta \dot{\xi}-6 H \delta \xi)+2 \xi \dot{\phi} \kappa-2 \dot{\phi}(2 \dot{\xi}-9 H \xi) \alpha\right]
\end{aligned}
$$

\footnotetext{
${ }^{1}$ Another forms can be found in eqs. (5-7) of [21] with a couple of minor typos in eq. (5): these are the fourth term in fourth line $\left(\phi_{\sigma \tau} \rightarrow \phi_{; \sigma \tau}\right)$ and the sixth term in fifth line $\left(\xi \delta_{\nu}^{\mu} \rightarrow \delta_{\nu}^{\mu}\right)$.

${ }^{2}$ Although eqs. $(97,98)$ were not presented in [21], these were derived together with Cyril Cartier while preparing [21].
} 


$$
\begin{aligned}
& +\frac{3}{2} c_{4} \dot{\phi}^{3}(4 \xi \delta \dot{\phi}+\dot{\phi} \delta \xi-4 \xi \dot{\phi} \alpha) \\
& \delta T_{\alpha}^{(c) \alpha}=4 c_{1}\left[-3 H^{2} \delta \ddot{\xi}-6 H\left(\dot{H}+H^{2}\right) \delta \dot{\xi}+2\left(\dot{H}+H^{2}\right) \frac{\Delta}{a^{2}} \delta \xi+2 H \dot{\xi} \dot{\kappa}\right. \\
& \left.+2\left(H \ddot{\xi}+\dot{H} \dot{\xi}+3 H^{2} \dot{\xi}\right) \kappa+3 H^{2} \dot{\xi} \dot{\alpha}+6 H\left(H \ddot{\xi}+2 \dot{H} \dot{\xi}+H^{2} \dot{\xi}\right) \alpha+2 \frac{\Delta}{a^{2}}(\ddot{\xi} \varphi+H \dot{\xi} \alpha)\right] \\
& +c_{2}\left\{6 H \xi \dot{\phi} \delta \ddot{\phi}+3\left(2 \dot{H} \xi \dot{\phi}+2 H \xi \ddot{\phi}+3 H^{2} \xi \dot{\phi}+2 H \dot{\xi} \dot{\phi}\right) \delta \dot{\phi}+3 H \dot{\phi}^{2} \delta \dot{\xi}\right. \\
& +\frac{3}{2} \dot{\phi}\left(2 \dot{H} \dot{\phi}+4 H \ddot{\phi}+3 H^{2} \dot{\phi}\right) \delta \xi-\xi \dot{\phi}^{2} \dot{\kappa}-\dot{\phi}(\dot{\xi} \dot{\phi}+2 \xi \ddot{\phi}+3 H \xi \dot{\phi}) \kappa-6 H \xi \dot{\phi}^{2} \dot{\alpha} \\
& \left.-9 \dot{\phi}\left(\dot{H} \xi \dot{\phi}+2 H \xi \ddot{\phi}+H^{2} \xi \dot{\phi}+H \dot{\xi} \dot{\phi}\right) \alpha-\frac{\Delta}{a^{2}}\left[2(\dot{\xi} \dot{\phi}+\xi \ddot{\phi}+H \xi \dot{\phi}) \delta \phi-\dot{\phi}^{2} \delta \xi-\xi \dot{\phi}^{2}(\varphi-\alpha)\right]\right\} \\
& +\frac{3}{2} c_{3} \dot{\phi}\left[2 \xi \dot{\phi} \delta \ddot{\phi}+(3 \dot{\xi} \dot{\phi}+4 \xi \ddot{\phi}) \delta \dot{\phi}+\dot{\phi}^{2} \delta \dot{\xi}+2 \dot{\phi} \ddot{\phi} \delta \xi-2 \xi \dot{\phi}^{2} \dot{\alpha}-4\left(\dot{\xi} \dot{\phi}^{2}+2 \xi \dot{\phi} \ddot{\phi}\right) \alpha\right] \\
& -\frac{3}{2} c_{4} \dot{\phi}^{3}(4 \xi \delta \dot{\phi}+\dot{\phi} \delta \xi-4 \xi \dot{\phi} \alpha) \text {, } \\
& \delta T_{\alpha}^{(c) 0}=\frac{1}{a} \nabla_{\alpha}\left\{\frac{4}{3} c_{1} H\left[3 H \delta \dot{\xi}-3 H^{2} \delta \xi-2 \dot{\xi}\left(\kappa+\frac{\Delta}{a^{2}} \chi\right)-3 H \dot{\xi} \alpha\right]\right. \\
& +c_{2} \dot{\phi}\left[-2 H \xi \delta \dot{\phi}+3 H^{2} \xi \delta \phi-H \dot{\phi} \delta \xi+\frac{1}{3} \xi \dot{\phi}\left(\kappa+6 H \alpha+\frac{\Delta}{a^{2}} \chi\right)\right] \\
& \left.-\frac{1}{2} c_{3} \dot{\phi}^{2}[2 \xi \delta \dot{\phi}+(\dot{\xi}-6 H \xi) \delta \phi+\dot{\phi} \delta \xi-2 \xi \dot{\phi} \alpha]+2 c_{4} \xi \dot{\phi}^{3} \delta \phi\right\}, \\
& \delta T_{\beta}^{(c) \alpha}-\frac{1}{3} \delta_{\beta}^{\alpha} \delta T_{\gamma}^{(c) \gamma}=\frac{1}{a^{2}}\left(\nabla^{\alpha} \nabla_{\beta}-\frac{1}{3} \delta_{\beta}^{\alpha} \Delta\right)\left\{4 c_{1}\left[H \dot{\xi} \dot{\chi}+\left(H \ddot{\xi}+\dot{H} \dot{\xi}+H^{2} \dot{\xi}\right) \chi-\ddot{\xi} \varphi-H \dot{\xi} \alpha-\left(\dot{H}+H^{2}\right) \delta \xi\right]\right. \\
& \left.-\frac{1}{2} c_{2}\left[\dot{\phi}^{2} \delta \xi-2(\dot{\xi} \dot{\phi}+\xi \ddot{\phi}+H \xi \dot{\phi}) \delta \phi+\xi \dot{\phi}^{2}(\varphi-\alpha+\dot{\chi})+\dot{\phi}(\dot{\xi} \dot{\phi}+2 \xi \ddot{\phi}+H \xi \dot{\phi}) \chi\right]\right\} \\
& +4 c_{1}\left[\left(H \dot{\xi} \dot{C}_{\beta}^{\alpha}\right)+3 H^{2} \dot{\xi} \dot{C}_{\beta}^{\alpha}-\ddot{\xi} \frac{\Delta}{a^{2}} C_{\beta}^{\alpha}\right]-\frac{1}{2} c_{2}\left[\left(\xi \dot{\phi}^{2} \dot{C}_{\beta}^{\alpha}\right)+3 H \dot{\phi}^{2} \xi \dot{C}_{\beta}^{\alpha}+\xi \dot{\phi}^{2} \frac{\Delta}{a^{2}} C_{\beta}^{\alpha}\right], \\
& \delta T^{(c)}=c_{1}\left(\xi_{, \phi \phi} \bar{R}_{G B}^{2} \delta \phi+\xi_{, \phi} \delta R_{G B}^{2}\right) \\
& +c_{2}\left\{-\left[6 H^{2} \xi \delta \ddot{\phi}+3 H\left(4 \dot{H} \xi+H \dot{\xi}+6 H^{2} \xi\right) \delta \dot{\phi}-2\left(2 \dot{H}+3 H^{2}\right) \xi \frac{\Delta}{a^{2}} \delta \phi\right]\right. \\
& -3 H\left[H \dot{\phi} \delta \dot{\xi}+2\left(H \ddot{\phi}+2 \dot{H} \dot{\phi}+3 H^{2} \dot{\phi}\right) \delta \xi\right]+2 \frac{\Delta}{a^{2}}[2 H \xi \dot{\phi} \alpha+(\dot{\xi} \dot{\phi}+2 \xi \ddot{\phi}+2 H \xi \dot{\phi}) \varphi] \\
& +2 \xi\left[2 H \dot{\phi} \dot{\kappa}+\left(2 H \ddot{\phi}+2 \dot{H} \dot{\phi}+9 H^{2} \dot{\phi}\right) \kappa\right]+2 H \dot{\xi} \dot{\phi}(\kappa+3 H \alpha) \\
& \left.+6 H \xi\left[H \dot{\phi} \dot{\alpha}+\left(2 H \ddot{\phi}+4 \dot{H} \dot{\phi}+3 H^{2} \dot{\phi}\right) \alpha\right]\right\} \\
& +c_{3}\left\{3 \dot{\phi}(\dot{\xi}-4 H \xi) \delta \ddot{\phi}+\left[2 \ddot{\xi} \dot{\phi}+3 \dot{\xi} \ddot{\phi}-12 \xi\left(3 H^{2} \dot{\phi}+\dot{H} \dot{\phi}+H \ddot{\phi}\right)\right] \delta \dot{\phi}\right. \\
& +[\dot{\xi} \dot{\phi}+4 \xi(\ddot{\phi}+2 \dot{\phi} H)] \frac{\Delta}{a^{2}} \delta \phi \\
& +\dot{\phi}\left[\dot{\phi} \delta \ddot{\xi}+3 \ddot{\phi} \delta \dot{\xi}-6\left(2 H \ddot{\phi}+\dot{H} \dot{\phi}+3 H^{2} \dot{\phi}\right) \delta \xi-\dot{\phi} \frac{\Delta}{a^{2}} \delta \xi\right]+2 \xi \dot{\phi}[\dot{\phi} \dot{\kappa}+2 \kappa(\ddot{\phi}+3 H \dot{\phi})]
\end{aligned}
$$




$$
\begin{aligned}
& \left.-4 \dot{\phi}^{2} \dot{\alpha}(\dot{\xi}-3 \xi H)-4 \ddot{\xi}^{2} \alpha-12 \dot{\xi} \dot{\phi} \ddot{\phi} \alpha+2 \xi \dot{\phi}\left[9\left(2 H \ddot{\phi}+\dot{H} \dot{\phi}+2 H^{2} \dot{\phi}\right)+\dot{\phi} \frac{\Delta}{a^{2}}\right] \alpha\right\} \\
& +c_{4}\left\{-\dot{\phi}\left[12 \xi \dot{\phi} \delta \ddot{\phi}+3(3 \dot{\xi} \dot{\phi}+8 \dot{\phi} \ddot{\phi}+12 H \xi \dot{\phi}) \delta \dot{\phi}-4 \xi \dot{\phi} \frac{\Delta}{a^{2}} \delta \phi\right]+4 \xi \dot{\phi}^{3}(\kappa+3 \dot{\alpha})\right. \\
& \left.-3 \dot{\phi}^{2}[\dot{\phi} \delta \dot{\xi}+4(\ddot{\phi}+H \dot{\phi}) \delta \xi]+12 \alpha \dot{\phi}^{2}[\dot{\xi} \dot{\phi}+\xi(4 \ddot{\phi}+3 H \dot{\phi})]\right\},
\end{aligned}
$$

where

$$
\bar{R}_{G B}^{2}=24 H^{2}\left(\dot{H}+H^{2}\right), \quad \delta R_{G B}^{2}=4 H^{2} \delta R-16 \dot{H}\left(H \kappa+\frac{\Delta}{a^{2}} \varphi\right),
$$

and $R$ and $\delta R$ are given in eqs. (51,52).

In this and next subsections, for simplicity, we assume $K=0$; we believe it is possible, perhaps tedious though, to derive corresponding equations for general $K$ in similar forms. We introduce

$$
\Phi \equiv \varphi_{\delta \phi}, \quad \Psi \equiv \varphi_{\chi}+\frac{\dot{F}+Q_{a}}{2 F+Q_{b}} \frac{\delta F_{\chi}}{\dot{F}} .
$$

From eqs. (13-15) and eqs. (13,15,16), respectively, we have

$$
\begin{aligned}
& \dot{\Phi}=2 \frac{\left(H+\frac{\dot{F}+Q_{a}}{2 F+Q_{b}}\right)\left(F+\frac{1}{2} Q_{b}\right)}{\omega \dot{\phi}^{2}+3 \frac{\left(\dot{F}+Q_{a}\right)^{2}}{2 F+Q_{b}}+Q_{c}} \frac{\Delta}{a^{2}} \Psi, \\
& \frac{H+\frac{\dot{F}+Q_{a}}{2 F+Q_{b}}}{a\left(F+\frac{1}{2} Q_{b}\right)}\left[\frac{a\left(F+\frac{1}{2} Q_{b}\right)}{H+\frac{\dot{F}+Q_{a}}{2 F+Q_{b}}} \Psi\right]=\frac{1}{2} \frac{\omega \dot{\phi}^{2}+3 \frac{\left(\dot{F}+Q_{a}\right)^{2}}{2 F+Q_{b}}+Q_{c}+Q_{d}+\frac{\dot{F}+Q_{a}}{2 F+Q_{b}} Q_{e}+\left(\frac{\dot{F}+Q_{a}}{2 F+Q_{b}}\right)^{2} Q_{f}}{\left(H+\frac{\dot{F}+Q_{a}}{2 F+Q_{b}}\right)\left(F+\frac{1}{2} Q_{b}\right)} \Phi,
\end{aligned}
$$

where

$$
\begin{aligned}
Q_{a} & \equiv-4 c_{1} \dot{\xi} H^{2}+2 c_{2} \xi \dot{\phi}^{2} H+c_{3} \xi \dot{\phi}^{3}, \\
Q_{b} & \equiv-8 c_{1} \dot{\xi} H+c_{2} \xi \dot{\phi}^{2}, \\
Q_{c} & \equiv-3 c_{2} \xi \dot{\phi}^{2} H^{2}+2 c_{3} \dot{\phi}^{3}(\dot{\xi}-3 \xi H)-6 c_{4} \xi \dot{\phi}^{4}, \\
Q_{d} & \equiv-2 c_{2} \xi \dot{\phi}^{2} \dot{H}-2 c_{3} \dot{\phi}^{2}(\dot{\xi} \dot{\phi}+\xi \ddot{\phi}-\xi \dot{\phi} H)+4 c_{4} \xi \dot{\phi}^{4}, \\
Q_{e} & \equiv-16 c_{1} \dot{\xi} \dot{H}+2 c_{2} \dot{\phi}(\dot{\xi} \dot{\phi}+2 \xi \ddot{\phi}-2 \xi \dot{\phi} H)-4 c_{3} \xi \dot{\phi}^{3}, \\
Q_{f} & \equiv 8 c_{1}(\ddot{\xi}-\dot{\xi} H)+2 c_{2} \xi \dot{\phi}^{2} .
\end{aligned}
$$

By combining eqs. $(101,102)$ we have

$$
\begin{aligned}
& \frac{\left(H+\frac{\dot{F}+Q_{a}}{2 F+Q_{b}}\right)^{2}}{a^{3}\left(\omega \dot{\phi}^{2}+3 \frac{\left(\dot{F}+Q_{a}\right)^{2}}{2 F+Q_{b}}+Q_{c}\right)}\left[\frac{a^{3}\left(\omega \dot{\phi}^{2}+3 \frac{\left(\dot{F}+Q_{a}\right)^{2}}{2 F+Q_{b}}+Q_{c}\right)}{\left(H+\frac{\dot{F}+Q_{a}}{2 F+Q_{b}}\right)^{2}} \dot{\Phi}\right]=c_{A}^{2} \frac{\Delta}{a^{2}} \Phi \\
& \frac{\omega \dot{\phi}^{2}+3 \frac{\left(\dot{F}+Q_{a}\right)^{2}}{2 F+Q_{b}}+Q_{c}+Q_{d}+\frac{\dot{F}+Q_{a}}{2 F+Q_{b}} Q_{e}+\left(\frac{\dot{F}+Q_{a}}{2 F+Q_{b}}\right)^{2} Q_{f}}{\left(H+\frac{\dot{F}+Q_{a}}{2 F+Q_{b}}\right)\left(F+\frac{1}{2} Q_{b}\right)} \\
& \times\left\{\frac{\left(H+\frac{\dot{F}+Q_{a}}{2 F+Q_{b}}\right)^{2}}{a\left[\omega \dot{\phi}^{2}+3 \frac{\left(\dot{F}+Q_{a}\right)^{2}}{2 F+Q_{b}}+Q_{c}+Q_{d}+\frac{\dot{F}+Q_{a}}{2 F+Q_{b}} Q_{e}+\left(\frac{\dot{F}+Q_{a}}{2 F+Q_{b}}\right)^{2} Q_{f}\right]}\left[\frac{a\left(F+\frac{1}{2} Q_{b}\right)}{H+\frac{\dot{F}+Q_{a}}{2 F+Q_{b}}} \Psi\right]=c_{A}^{2} \frac{\Delta}{a^{2}} \Psi,\right.
\end{aligned}
$$

where 


$$
c_{A}^{2}=1+\frac{Q_{d}+\frac{\dot{F}+Q_{a}}{2 F+Q_{b}} Q_{e}+\left(\frac{\dot{F}+Q_{a}}{2 F+Q_{b}}\right)^{2} Q_{f}}{\omega \dot{\phi}^{2}+3 \frac{\left(\dot{F}+Q_{a}\right)^{2}}{2 F+Q_{b}}+Q_{c}} .
$$

A closed form second-order equation in terms of $\Phi$ was derived in $[20,21]$. Using eq. (64) we have

$$
\varphi_{\chi}=\frac{H \Psi+\frac{\dot{F}+Q_{a}}{2 F+Q_{b}} \varphi_{\delta \phi}}{H+\frac{\dot{F}+Q_{a}}{2 F+Q_{b}}} .
$$

Notice that the presence of any $c_{i}$ terms affects $c_{A}$ in non-trivial ways.

For the tensor mode we have $[20,21]$

$$
\frac{1}{a^{3} Q_{t}}\left(a^{3} Q_{t} \dot{C}_{\alpha \beta}\right)^{\cdot}-c_{T}^{2} \frac{\Delta}{a^{2}} C_{\alpha \beta}=0,
$$

where

$$
Q_{t} \equiv F+\frac{1}{2} Q_{b}, \quad c_{T}^{2} \equiv 1-\frac{Q_{f}}{2 F+Q_{b}} .
$$

This equation is valid for general algebraic function of $f(\phi, R)$. From this wave equation we can read that $c_{T}$ has a role of the gravitational wave propagation speed. $c_{T}$ is affected by the presence of the $c_{1}$ and $c_{2}$ correction terms only.

\section{G. String axionic correction}

We consider an action in eq. (45) with the following additional correction term [22]

$$
L_{(c)}=\frac{1}{8} \nu(\phi) R \tilde{R}
$$

where $R \tilde{R} \equiv \eta^{a b c d} R_{a b}{ }^{e f} R_{c d e f}$ with $\eta^{a b c d}$ a totally antisymmetric Levi-Civita tensor density. Corrections to the gravitational field equation and the equation of motion are

$$
\begin{aligned}
& T_{a b}^{(c)}=\eta_{a}{ }^{c d e}\left(\nu_{, e ; f} R_{b c d}^{f}-2 \nu_{, e} R_{b c ; d}\right), \\
& T^{(c)}=-\frac{1}{4} \nu_{, \phi} R \tilde{R} .
\end{aligned}
$$

Assuming $K=0$, the only nonvanishing contribution is

$$
\begin{aligned}
& T_{\beta}^{(c) \alpha}=\frac{1}{a} \epsilon^{\alpha \gamma \delta}\left[(\ddot{\nu}-H \dot{\nu}) \dot{C}_{\beta \gamma, \delta}+\dot{\nu} D_{\beta \gamma, \delta}\right]+(\alpha \leftrightarrow \beta), \\
& T^{(c)}=0
\end{aligned}
$$

where

$$
D_{\alpha \beta} \equiv \ddot{C}_{\alpha \beta}+3 H \dot{C}_{\alpha \beta}-\frac{\Delta}{a^{2}} C_{\alpha \beta},
$$

and we introduced $\epsilon^{\alpha \beta \gamma} \equiv a^{4} \bar{\eta}^{0 \alpha \beta \gamma}$ which is based on $g_{\alpha \beta}^{(3)}$. Thus, the string axion correction term $\nu R \tilde{R}$ does not affect the background equations nor the scalar-type perturbation. Thus, eqs. (57-65) in the generalized $f(\phi, R)$ gravity remain valid. It affects, however, the tensor mode, and the $(\alpha, \beta)$-component of the field equation, or eqs. $(20,50,53)$, give

$$
\frac{1}{a^{3} F}\left(a^{3} F \dot{C}_{\alpha \beta}\right)^{\cdot}-\frac{\Delta}{a^{2}} C_{\alpha \beta}-\frac{2}{a F} \epsilon_{(\alpha}^{\gamma \delta}\left[(\ddot{\nu}-H \dot{\nu}) \dot{C}_{\beta) \gamma}+\dot{\nu} D_{\beta) \gamma}\right]_{, \delta}=0 .
$$

This equation is more general than the one derived in [22]; it includes our generalized gravity coupling $f(\phi, R)$ in its most general algebraic form. We expand $[23,26]$ 


$$
C_{\alpha \beta}(\mathbf{x}, t) \equiv \sqrt{\operatorname{Vol}} \int \frac{d^{3} k}{(2 \pi)^{3}} \sum_{\ell} e_{\alpha \beta}^{(\ell)}(\mathbf{k}) h_{\ell \mathbf{k}}(t) e^{i \mathbf{k} \cdot \mathbf{x}},
$$

where $e_{\alpha \beta}^{(\ell)}$ is the circular polarization tensor $(\ell=L, R)$ with the property $i k_{\gamma} \epsilon_{\alpha}^{\gamma \delta} e_{\beta \delta}^{(\ell)}=k \lambda_{\ell} e_{\alpha \beta}^{(\ell)}\left(\lambda_{L}=-1\right.$ and $\left.\lambda_{R}=+1\right)$. We have

$$
\frac{1}{a^{3} Q_{t}}\left(a^{3} Q_{t} \dot{h}_{\ell \mathbf{k}}\right)^{\cdot}+\frac{k^{2}}{a^{2}} h_{\ell \mathbf{k}}=0,
$$

where

$$
Q_{t} \equiv F+2 \lambda_{\ell} \dot{\nu} k / a .
$$

To make this equation similar to the other gravity theories we may set $\Phi \equiv h_{\ell \mathbf{k}}$. We notice that the presence of stringaxionic correction term leads to asymmetric generation and evolution of the two polarization states of gravitational wave. However, the wave propagation speed remains $c_{A}=1$.

\section{CLASSICAL EVOLUTION: UNIFIED FORM}

\section{A. Equations}

All the basic scalar-type perturbation equations considered in $\S$ III A-III G can be written in the following forms

$$
\begin{aligned}
& \dot{\Phi}=2 x_{1} \frac{\Delta}{a^{2}} \Psi, \\
& \frac{1}{x_{2}}\left(x_{2} \Psi\right)^{\cdot}=\frac{1}{2} x_{3} \Phi .
\end{aligned}
$$

Notice that in these forms the normalization of $x_{2}$ is arbitrary. In order to be consistent in unified form in the action formulation in eq. (138) we fix the normalization in the following way: we read $x_{2}$ directly from eqs. (59,86,102) whereas from eqs. $(24,40)$ we read $x_{2} \equiv \frac{1}{8 \pi G} \frac{a}{H}$. In Einstein's gravity limit we have $F=\frac{1}{8 \pi G}$. Introducing

$$
\bar{z} \equiv c_{A} z \equiv \sqrt{a x_{2} x_{3}}, \quad c_{A} \equiv \sqrt{x_{1} x_{3}} ; \quad \tilde{v} \equiv z \Phi, \quad u \equiv x_{2} \frac{1}{\bar{z}} \Psi
$$

we have

$$
\tilde{v}=\frac{2}{c_{A} \bar{z}}(\bar{z} u)^{\prime}, \quad u=\frac{1}{2} \Delta^{-1} \frac{z}{c_{A}}\left(\frac{\tilde{v}}{z}\right)^{\prime},
$$

where a prime indicates a time derivative based on $\eta$ with $d t \equiv a d \eta$. Thus, we have

$$
\begin{aligned}
& \tilde{v}^{\prime \prime}-\left(c_{A}^{2} \Delta+\frac{z^{\prime \prime}}{z}\right) \tilde{v}=a^{2} z\left[\frac{1}{a z^{2}}\left(a z^{2} \dot{\Phi}\right)-c_{A}^{2} \frac{\Delta}{a^{2}} \Phi\right]=0 \\
& u^{\prime \prime}-\left[c_{A}^{2} \Delta+\frac{(1 / \bar{z})^{\prime \prime}}{(1 / \bar{z})}\right] u=\frac{a^{2} x_{2}}{\bar{z}}\left\{\frac{\bar{z}^{2}}{a x_{2}}\left[\frac{a}{\bar{z}^{2}}\left(x_{2} \Psi\right)^{\cdot}\right]-c_{A}^{2} \frac{\Delta}{a^{2}} \Psi\right\}=0 .
\end{aligned}
$$

In these forms of the wave equation, $c_{A}$ has the role of wave speed of the fluctuating fluid or field and the simultaneously excited metric. For convenience, we summarize various coefficients in Table 1.

For the tensor mode, using

[ TABLE 1]]

$$
z_{t} \equiv a \sqrt{Q_{t}}, \quad v_{t} \equiv z_{t} \Phi
$$

with $\Phi=C_{\alpha \beta}$ or $h_{\ell \mathbf{k}}$, we have

$$
v_{t}^{\prime \prime}-\left(c_{T}^{2} \Delta+\frac{z_{t}^{\prime \prime}}{z_{t}}\right) v_{t}=a^{2} z_{t}\left[\frac{1}{a z_{t}^{2}}\left(a z_{t}^{2} \dot{\Phi}\right)-c_{T}^{2} \frac{\Delta}{a^{2}} \Phi\right]=0 .
$$

Thus, it can be absorbed to eq. (124) as a unified form. We summarize various coefficients in the gravitational wave in Table 2.

[[TABLE 2]]

Equations $(124,127)$ in the context of Einstein's gravity are the starting point of diverse analyses in the context of inflationary structure generation based on quantum fluctuations. In this work we have shown that these equations are generally valid in a wide variety of gravity theories we are considering. 


\section{B. Solutions}

We have the following general solutions. We introduce the Fourier transformations of perturbation variables. Since we are considering linear order perturbation, the equations of the Fourier transformed variables satisfy the same equations as in the original configuration space with $\Delta=-k^{2}$. Thus, we do not distinguish explicitly the Fourier variables from the original ones.

1. In the large-scale limits, with $c_{A}^{2} k^{2} \ll z^{\prime \prime} / z$ and $(1 / \bar{z})^{\prime \prime} /(1 / \bar{z})$, we have

$$
\begin{aligned}
& \Phi(k, \eta)=\frac{1}{z} \tilde{v}=C(k)\left\{1+k^{2}\left[\int^{\eta} \bar{z}^{2}\left(\int^{\eta} \frac{d \eta}{z^{2}}\right) d \eta-\int^{\eta} \bar{z}^{2} d \eta \int^{\eta} \frac{d \eta}{z^{2}}\right]\right\}-2 \tilde{d}(k) k^{2} \int^{\eta} \frac{d \eta}{z^{2}} \\
& \Psi(k, \eta)=\frac{\bar{z}}{x_{2}} u=C(k) \frac{1}{2 x_{2}} \int^{\eta} \bar{z}^{2} d \eta+\tilde{d}(k) \frac{1}{x_{2}}\left\{1+k^{2}\left[\int^{\eta} \frac{1}{z^{2}}\left(\int^{\eta} \bar{z}^{2} d \eta\right) d \eta-\int^{\eta} \bar{z}^{2} d \eta \int^{\eta} \frac{d \eta}{z^{2}}\right]\right\}
\end{aligned}
$$

The $C(d)$-mode is relatively growing (decaying) in the expanding phase of the background world model ${ }^{3}$. Notice that to the leading order in the large-scale expansion the $C$-mode of $\Phi$ remains constant whereas the one of $\Psi$ changes its behavior according to the background evolution. Thus, ignoring the transient mode we have

$$
\Phi(k, \eta)=C(k)
$$

It is remarkable that

"the constant nature of $\Phi$ in the expanding phase is valid independently of changing (i) equation of state $p(\mu)$, (ii) field potential $V(\phi)$, and (iii) gravity theories $f(\phi, R, X), \omega(\phi), \xi(\phi)$ and $\nu(\phi) . "$

2. In the small-scale limits, with $c_{A}^{2} k^{2} \gg z^{\prime \prime} / z$ and $(1 / \bar{z})^{\prime \prime} /(1 / \bar{z})$, we have

$$
\begin{aligned}
& \tilde{v}(k, \eta)=z \Phi=c_{v_{1}} e^{i c_{A} k \eta}+c_{v_{2}} e^{-i c_{A} k \eta}, \\
& u(k, \eta)=\frac{x_{2}}{\bar{z}} \Psi=\frac{i}{2 k}\left(-c_{v_{1}} e^{i c_{A} k \eta}+c_{v_{2}} e^{-i c_{A} k \eta}\right),
\end{aligned}
$$

where we assumed $c_{A}=$ constant.

Although expressed in general forms, considering the complications in $c_{A}^{2}$ for the field and generalized gravities, these solutions in 1. and 2. are properly applicable for $K=0$.

3. We have exact solutions under

$$
z \propto|\eta|^{q}, \quad c_{A}^{2}=\text { constant. }
$$

We have

$$
\frac{z^{\prime \prime}}{z}=\frac{q(q-1)}{\eta^{2}} \equiv \frac{n}{\eta^{2}}
$$

and exact solutions are

$$
\begin{aligned}
& \Phi(k, \eta)=\frac{\sqrt{\pi|\eta|}}{2 z}\left[c_{1}(k) H_{\nu}^{(1)}\left(c_{A} k|\eta|\right)+c_{2}(k) H_{\nu}^{(2)}\left(c_{A} k|\eta|\right)\right] \\
& \Psi(k, \eta)=-\frac{\sqrt{\pi|\eta|}}{2 z} \frac{a c_{A}}{2 k x_{1}}\left[c_{1}(k) H_{\nu-1}^{(1)}\left(c_{A} k|\eta|\right)+c_{2}(k) H_{\nu-1}^{(2)}\left(c_{A} k|\eta|\right)\right]
\end{aligned}
$$

where

$$
\nu \equiv \frac{1}{2}-q=\sqrt{n+\frac{1}{4}} .
$$

The normalization is still arbitrary; see eq. (141) for a normalization assuming the vacuum expectation value.

According to the prescription in eq. (126) the above solution for $\Phi$ applies to the gravitational wave as well; see eq. (150) for proper normalization.

\footnotetext{
${ }^{3}$ The roles reverse in the collapsing phase. In a collapsing phase the $d$-mode rapidly grows and unambiguously becomes singular as the background approaches a singularity [27].
} 


\section{QUANTUM GENERATION: UNIFIED FORM}

\section{A. Quantization}

The perturbed action becomes $[28,13]$

$$
\begin{aligned}
\delta^{2} S & =\frac{1}{2} \int a z^{2}\left(\dot{\Phi}^{2}-c_{A}^{2} \frac{1}{a^{2}} \Phi^{, \alpha} \Phi, \alpha\right) d t d^{3} x \\
& =\frac{1}{2} \int\left(\tilde{v}^{\prime 2}-c_{A}^{2} \tilde{v}^{, \alpha} \tilde{v}_{, \alpha}+\frac{z^{\prime \prime}}{z} \tilde{v}^{2}\right) d \eta d^{3} x
\end{aligned}
$$

The mode expansion is

$$
\begin{aligned}
& \hat{\Phi}(\mathbf{x}, t) \equiv \int \frac{d^{3} k}{(2 \pi)^{3 / 2}}\left[\hat{a}_{\mathbf{k}} \Phi_{\mathbf{k}}(t) e^{i \mathbf{k} \cdot \mathbf{x}}+\hat{a}_{\mathbf{k}}^{\dagger} \Phi_{\mathbf{k}}^{*}(t) e^{-i \mathbf{k} \cdot \mathbf{x}}\right] \\
& {\left[\hat{a}_{\mathbf{k}}, \hat{a}_{\mathbf{k}^{\prime}}\right]=0, \quad\left[\hat{a}_{\mathbf{k}}^{\dagger}, \hat{a}_{\mathbf{k}^{\prime}}^{\dagger}\right]=0, \quad\left[\hat{a}_{\mathbf{k}}, \hat{a}_{\mathbf{k}^{\prime}}^{\dagger}\right]=\delta^{3}\left(\mathbf{k}-\mathbf{k}^{\prime}\right)}
\end{aligned}
$$

The conjugate momentum is $\pi_{\Phi} \equiv \frac{\partial \mathcal{L}}{\partial \dot{\Phi}}=a z^{2} \dot{\Phi}$. The quantization condition $\left[\hat{\Phi}(\mathbf{x}, t), \hat{\pi}_{\Phi}\left(\mathbf{x}^{\prime}, t\right)\right]=i \delta^{3}\left(\mathbf{x}-\mathbf{x}^{\prime}\right)$ gives $\left[\hat{\Phi}(\mathbf{x}, t), \dot{\hat{\Phi}}\left(\mathbf{x}^{\prime}, t\right)\right]=\frac{i}{a z^{2}} \delta^{3}\left(\mathbf{x}-\mathbf{x}^{\prime}\right)$ which leads to the Wronskian condition

$$
\Phi_{\mathbf{k}} \dot{\Phi}_{\mathbf{k}}^{*}-\Phi_{\mathbf{k}}^{*} \dot{\Phi}_{\mathbf{k}}=\frac{i}{a z^{2}}
$$

If the background satisfies eq. (133) we have eqs. $(135,136)$ as exact solutions. In terms of the mode function we have $[14,24]$

$$
\begin{aligned}
& \Phi_{\mathbf{k}}(\eta)=\frac{\sqrt{\pi|\eta|}}{2 z}\left[c_{1}(k) H_{\nu}^{(1)}\left(c_{A} k|\eta|\right)+c_{2}(k) H_{\nu}^{(2)}\left(c_{A} k|\eta|\right)\right], \\
& \Psi_{\mathbf{k}}(\eta)=-\frac{\sqrt{\pi|\eta|}}{2 z} \frac{a c_{A}}{2 k x_{1}}\left[c_{1}(k) H_{\nu-1}^{(1)}\left(c_{A} k|\eta|\right)+c_{2}(k) H_{\nu-1}^{(2)}\left(c_{A} k|\eta|\right)\right]
\end{aligned}
$$

where

$$
\left|c_{2}(k)\right|^{2}-\left|c_{1}(k)\right|^{2}=1
$$

which follows from the quantization condition in eq. (140). The two-point function is defined as $[25,14]$

$$
G_{\Phi}\left(x^{\prime}, x^{\prime \prime}\right) \equiv\left\langle\hat{\Phi}\left(x^{\prime}\right) \hat{\Phi}\left(x^{\prime \prime}\right)\right\rangle_{\mathrm{vac}}=\int_{0}^{\infty} \frac{k^{2} d k}{2 \pi^{2}} j_{0}\left(k\left|\mathbf{x}^{\prime}-\mathbf{x}^{\prime \prime}\right|\right) \Phi_{\mathbf{k}}\left(\eta^{\prime}\right) \Phi_{\mathbf{k}}^{*}\left(\eta^{\prime \prime}\right)
$$

where \langle\rangle$_{\text {vac }}$ is a vacuum expectation value with $\hat{a}_{\mathbf{k}}|\mathrm{vac}\rangle \equiv 0$ for every $\mathbf{k} ; x=(\mathbf{x}, \eta)$. Using eq. (141) as the mode function solution, and assuming the simple vacuum state $c_{2}=1$ and $c_{1}=0$, we have $[14,24]$

$$
G_{\Phi}\left(x^{\prime}, x^{\prime \prime}\right)=\frac{\left(\frac{1}{4}-\nu^{2}\right) \sec (\pi \nu)}{16 \pi \eta^{\prime} \eta^{\prime \prime} z^{\prime} z^{\prime \prime}} F\left(\frac{3}{2}+\nu ; \frac{3}{2}-\nu ; 2 ; 1+\frac{\Delta \eta^{2}-\Delta \mathbf{x}^{2}}{4 \eta^{\prime} \eta^{\prime \prime}}\right),
$$

which is valid for $\nu<\frac{3}{2}$ and $\Delta \eta^{2}-\Delta \mathbf{x}^{2}<0 ; \Delta \eta^{2} \equiv\left(\eta^{\prime}-\eta^{\prime \prime}\right)^{2}$ and $\Delta \mathbf{x}^{2} \equiv\left(\mathbf{x}^{\prime}-\mathbf{x}^{\prime \prime}\right)^{2}$.

\section{B. Power spectra}

The seed generation process involves three steps.

1. We evaluate the power-spectrum based on a vacuum expectation value introduced as

$$
\mathcal{P}_{\hat{\Phi}}(k, t) \equiv \frac{k^{3}}{2 \pi^{2}} \int\langle\hat{\Phi}(\mathbf{x}+\mathbf{r}, t) \hat{\Phi}(\mathbf{x}, t)\rangle_{\mathrm{vac}} e^{-i \mathbf{k} \cdot \mathbf{r}} d^{3} r=\frac{k^{3}}{2 \pi^{2}}\left|\Phi_{k}(t)\right|^{2}
$$

In the large-scale limit using the mode-function solution in eq. (141) we have 


$$
\mathcal{P}_{\hat{\Phi}}^{1 / 2}(\mathbf{k}, \eta)=\frac{H}{2 \pi} \frac{1}{a H|\eta|} \frac{\Gamma(\nu)}{\Gamma(3 / 2)}\left(\frac{k|\eta|}{2}\right)^{3 / 2-\nu} \frac{1}{c_{A}^{\nu} z / a} .
$$

To include the general vacuum dependence we should multiply for the scalar-type perturbation

$$
\left|c_{2}(\mathbf{k})-c_{1}(\mathbf{k})\right|
$$

where

$$
\left|c_{2}(\mathbf{k})\right|^{2}-\left|c_{1}(\mathbf{k})\right|^{2}=1 .
$$

For $\nu=0$ we we have additional $2 \ln \left(c_{A} k|\eta|\right)$ factor.

For the tensor-type perturbation we have $\hat{\Phi}=\hat{C}_{\beta}^{\alpha}$ and we need additional $\sqrt{2}$ factor [26], with $c_{T}$ replacing $c_{A}$, thus

$$
\mathcal{P}_{\hat{C}_{\alpha \beta}}^{1 / 2}(\mathbf{k}, \eta)=\sqrt{16 \pi G} \frac{H}{2 \pi} \frac{1}{a H|\eta|} \frac{\Gamma\left(\nu_{t}\right)}{\Gamma(3 / 2)}\left(\frac{k|\eta|}{2}\right)^{3 / 2-\nu_{t}} \frac{1 / \sqrt{8 \pi G}}{c_{T}^{\nu_{t}} z_{t} / a} .
$$

To include the general vacuum dependence we should multiply

$$
\sqrt{\frac{1}{2} \sum_{\ell}\left|c_{\ell 2}(\mathbf{k})-c_{\ell 1}(\mathbf{k})\right|^{2}}
$$

where

$$
\left|c_{\ell 2}(\mathbf{k})\right|^{2}-\left|c_{\ell 1}(\mathbf{k})\right|^{2}=1 .
$$

$\ell=+, \times$ indicate two polarization states. For $\nu=0$ we we have additional $2 \ln \left(c_{A} k|\eta|\right)$ factor.

In the case of string axionic correction, which will affect only the gravitational wave, we need to handle the case separately. We have

$$
\mathcal{P}_{\hat{C}_{\alpha \beta}}^{1 / 2}(\mathbf{k}, \eta)=\sqrt{16 \pi G} \frac{H}{2 \pi} \frac{1}{a H|\eta|} \frac{\Gamma\left(\nu_{t}\right)}{\Gamma(3 / 2)}\left(\frac{k|\eta|}{2}\right)^{3 / 2-\nu_{t}} \frac{1}{c_{T}^{\nu_{t}}} \sqrt{\frac{1}{2} \sum_{\ell} \frac{1 / 8 \pi G}{\left|F+2 \lambda_{\ell} \dot{\nu} \frac{k}{a}\right|}\left|c_{\ell 2}(\mathbf{k})-c_{\ell 1}(\mathbf{k})\right|^{2}} .
$$

2. In the super-horizon scale we identify [29]

$$
\mathcal{P}_{\hat{\Phi}} \equiv \mathcal{P}_{\Phi}
$$

where

$$
\mathcal{P}_{\Phi}(k, t) \equiv \frac{k^{3}}{2 \pi^{2}} \int\langle\Phi(\mathbf{x}+\mathbf{r}, t) \Phi(\mathbf{x}, t)\rangle_{\mathbf{x}} e^{-i \mathbf{k} \cdot \mathbf{r}} d^{3} r=\frac{k^{3}}{2 \pi^{2}}|\Phi(k, t)|^{2},
$$

is a power-spectrum based on the spatial averaging. Compare the similarity between eqs. $(146,155)$.

3. The growing modes of $\Phi$ are conserved while in the large scale limit. Thus, the final classical power spectra of the large-scale structure and the gravitational wave $\mathcal{P}_{\Phi}$ is the same as the $\mathcal{P}_{\hat{\Phi}}$ generated from the quantum fluctuations in the early universe.

Spectral indices are defined as

$$
n_{S}-1, n_{T} \equiv \frac{\partial \ln \mathcal{P}_{\Phi}}{\partial \ln k}
$$

thus

$$
\mathcal{P}_{\Phi} \propto k^{n_{S}-1}, k^{n_{T}} .
$$

Assuming the simplest vacuum state, i.e., $c_{2}=1$ and $c_{1}=0$, etc., we have

$$
n_{S}-1, n_{T}=3-2 \nu=2+2 q .
$$

In the case of near Harrison-Zel'dovich spectra $\left(n_{S}-1 \simeq 0 \simeq n_{T}\right)$ the quadrupole anisotropy of the CMB becomes 


$$
\left\langle a_{2}^{2}\right\rangle=\left\langle a_{2}^{2}\right\rangle_{S}+\left\langle a_{2}^{2}\right\rangle_{T}=\frac{\pi}{75} \mathcal{P}_{\varphi_{\delta \phi}}+7.74 \frac{1}{5} \frac{3}{32} \mathcal{P}_{C_{\alpha \beta}},
$$

which is valid for $K=0=\Lambda$; for a general situation with nonvanishing $\Lambda$ we need numerical treatment, see [30]. The ratio between two types of perturbations is

$$
r_{2} \equiv \frac{\left\langle a_{2}^{2}\right\rangle_{T}}{\left\langle a_{2}^{2}\right\rangle_{S}} \simeq 3.46 \frac{\mathcal{P}_{C_{\alpha \beta}}}{\mathcal{P}_{\varphi_{\delta \phi}}}
$$

From eqs. $(147,150)$ we have

$$
r_{1} \equiv \frac{\mathcal{P}_{C_{\alpha \beta}}}{\mathcal{P}_{\varphi_{\delta \phi}}}=2\left[\left(\frac{k|\eta|}{2}\right)^{\nu-\nu_{t}} \frac{\Gamma\left(\nu_{t}\right)}{\Gamma(\nu)} \frac{c_{A}^{\nu-1}}{c_{T}^{\nu_{t}}} \frac{\bar{z}}{z_{t}}\right]^{2}
$$

Therefore, if the background evolution during the quantum generation stage satisfies eq. (133) we can read the power spectra (both scalar- and tensor-types) using eqs. $(146,141)$. In the large-scale limit we have the power spectra in eqs. $(147,150)$. The spectral indices (slopes) and the ratio of amplitudes are presented in eqs. (158,161). The contribution to the quadrupole angular anisotropy can be estimated using eq. (159). We would like to emphasize that all our results in $\S \mathrm{IV}$ and $\mathrm{V}$ are generally valid in our generalized gravity theories in unified forms.

The four-year COBE [31] data with a $n=1$ power-law fit give

$$
\begin{aligned}
& Q_{r m s-\left.P S\right|_{n=1}}=18 \pm 1.6 \mu K, \quad T=2.725 \pm 0.020 K \\
& \left\langle a_{2}^{2}\right\rangle=\frac{4 \pi}{5}\left(\frac{Q_{r m s-\left.P S\right|_{n=1}}}{T}\right)^{2} \simeq 1.1 \times 10^{-10}
\end{aligned}
$$

The observed quadrupole amplitude is $Q_{r m s}=10_{-4}^{+7} \mu K$ which is lower than the above fitted value. The first-year WMAP [32] data show even lower value of $Q_{r m s}=8 \pm 2 \mu K$ and the temperature $T=2.725 \pm 0.002 K$. WMAP data also provided a constraint on $r_{2}: r \equiv 4 \mathcal{P}_{C_{\alpha \beta}} / \mathcal{P}_{\varphi_{\delta \phi}}<0.90$ with $95 \%$ confidence and $n_{S}=0.99 \pm 0.04,[32]$.

\section{Slow-roll}

In the context of $f(\phi, R)$ gravity we have introduced the following parameters [17]

$$
\epsilon_{1} \equiv \frac{\dot{H}}{H^{2}}, \quad \epsilon_{2} \equiv \frac{\ddot{\phi}}{H \dot{\phi}}, \quad \epsilon_{3} \equiv \frac{1}{2} \frac{\dot{F}}{H F}, \quad \epsilon_{4} \equiv \frac{1}{2} \frac{\dot{E}}{H E} .
$$

$\epsilon_{1}$ and $\epsilon_{2}$ are the slow-roll parameters used in the minimally coupled scalar field, [33,34]. The two additional functional degrees of freedom in $F(\phi)$ and $\omega(\phi)$ are reflected in $\epsilon_{3}$ and $\epsilon_{4}$. In the context of string correction we have an additional functional degree of freedom in $\xi(\phi)$. In order to consider its effect we introduce the following additional parameters

$$
\epsilon_{5} \equiv \frac{\dot{F}+Q_{a}}{H\left(2 F+Q_{b}\right)}, \quad \epsilon_{6} \equiv \frac{\dot{Q}_{t}}{2 H Q_{t}}
$$

with

$$
E \equiv \frac{F}{\dot{\phi}^{2}}\left(\omega \dot{\phi}^{2}+3 \frac{\left(\dot{F}+Q_{a}\right)^{2}}{2 F+Q_{b}}+Q_{c}\right) .
$$

In the $f(\phi, R)$ gravity we have $\epsilon_{5}=\epsilon_{6}=\epsilon_{3}$, and $E$ in eq. (165) becomes the one in eq. (60). In the case of tachyonic corrections we introduce

$$
E \equiv-\frac{F}{2 X}\left(X f_{, X}+2 X^{2} f_{, X X}+\frac{3 \dot{F}^{2}}{2 F}\right)
$$

and take $\epsilon_{5}=\epsilon_{6}=\epsilon_{3}$.

Using the above definitions and eq. (122), assuming $K=0, z$ and $z_{t}$ can be written in unified forms 


$$
z \equiv \frac{a \dot{\phi} / H}{1+\epsilon_{5}} \sqrt{\frac{E}{F}}, \quad z_{t} \equiv a \sqrt{Q_{t}}
$$

Thus, we can derive

$$
\begin{aligned}
\frac{z^{\prime \prime}}{z}= & a^{2}\left[H^{2}\left(1-\epsilon_{1}+\epsilon_{2}-\epsilon_{3}+\epsilon_{4}\right)\left(2+\epsilon_{2}-\epsilon_{3}+\epsilon_{4}\right)+H\left(-\dot{\epsilon}_{1}+\dot{\epsilon}_{2}-\dot{\epsilon}_{3}+\dot{\epsilon}_{4}\right)\right. \\
& \left.-2\left(\frac{3}{2}-\epsilon_{1}+\epsilon_{2}-\epsilon_{3}+\epsilon_{4}\right) H \frac{\dot{\epsilon}_{5}}{1+\epsilon_{5}}-\frac{\ddot{\epsilon}_{5}}{1+\epsilon_{5}}+2 \frac{\dot{\epsilon}_{5}^{2}}{\left(1+\epsilon_{5}\right)^{2}}\right], \\
\frac{z_{t}^{\prime \prime}}{z_{t}}= & a^{2} H^{2}\left[\left(1+\epsilon_{6}\right)\left(2+\epsilon_{1}+\epsilon_{6}\right)+\frac{1}{H} \dot{\epsilon}_{6}\right] .
\end{aligned}
$$

Although we have introduced $\epsilon_{i} \mathrm{~S}$ as extension of slow-roll parameters, it is far from certain that these parameters can be properly called the slow-roll parameters. Thus, it is better to regard $\epsilon_{i} \mathrm{~S}$ as new definitions of the fundamental parameters $V(\phi), \omega(\phi), f(\phi, R)$, etc. If $\dot{\epsilon}_{1}=0$, we have

$$
\eta=-\frac{1}{a H} \frac{1}{1+\epsilon_{1}} .
$$

Thus, for $\dot{\epsilon}_{i}=0$ eqs. $(168,169)$ become

$$
\begin{aligned}
& \frac{z^{\prime \prime}}{z}=\frac{1}{\eta^{2}} \frac{1}{\left(1+\epsilon_{1}\right)^{2}}\left(1-\epsilon_{1}+\epsilon_{2}-\epsilon_{3}+\epsilon_{4}\right)\left(2+\epsilon_{2}-\epsilon_{3}+\epsilon_{4}\right) \equiv \frac{n_{s}}{\eta^{2}}, \\
& \frac{z_{t}^{\prime \prime}}{z_{t}}=\frac{1}{\eta^{2}} \frac{\left(1+\epsilon_{6}\right)\left(2+\epsilon_{1}+\epsilon_{6}\right)}{\left(1+\epsilon_{1}\right)^{2}} \equiv \frac{n_{t}}{\eta^{2}} .
\end{aligned}
$$

The spectral indices become ${ }^{4}$

$$
\begin{aligned}
& n_{S}-1=3-2 \nu=3-\sqrt{4 n_{s}+1}=2 \frac{2 \epsilon_{1}-\epsilon_{2}+\epsilon_{3}-\epsilon_{4}}{1+\epsilon_{1}}, \\
& n_{T}=3-2 \nu_{t}=3-\sqrt{4 n_{t}+1}=2 \frac{\epsilon_{1}-\epsilon_{6}}{1+\epsilon_{1}} .
\end{aligned}
$$

If $\epsilon_{1,2,3,4} \ll 1$ we have Harrison-Zel'dovich spectrum for the scalar-type perturbation, and if $\epsilon_{1,6} \ll 1$ we have the corresponding one for the tensor-type perturbation. In this case eqs. $(147,150)$ become

$$
\begin{aligned}
\mathcal{P}_{\hat{\Phi}}^{1 / 2}(\mathbf{k}, \eta) & =\left|\frac{H}{2 \pi}\left\{1+\epsilon_{1}+\left(2 \epsilon_{1}-\epsilon_{2}+\epsilon_{3}-\epsilon_{4}\right)\left[\ln (k|\eta|)-2+\ln 2+\gamma_{E}\right]\right\} \frac{1}{c_{A}^{\nu} z / a}\right|, \\
\mathcal{P}_{\hat{C}_{\alpha \beta}}^{1 / 2}(\mathbf{k}, \eta) & =\left|\sqrt{2} \frac{H}{2 \pi}\left\{1+\epsilon_{1}+\left(\epsilon_{1}-\epsilon_{6}\right)\left[\ln (k|\eta|)-2+\ln 2+\gamma_{E}\right]\right\} \frac{1}{c_{T}^{\nu_{t}} z_{t} / a}\right|,
\end{aligned}
$$

where $\gamma_{E}=0.57722$ is the Euler's constant. Thus,

$$
\begin{aligned}
& n_{S}-1=2\left(2 \epsilon_{1}-\epsilon_{2}+\epsilon_{3}-\epsilon_{4}\right), \quad n_{T}=2\left(\epsilon_{1}-\epsilon_{6}\right), \\
& r_{1} \equiv \frac{\mathcal{P}_{C_{\alpha \beta}}}{\mathcal{P}_{\varphi_{\delta \phi}}}=2\left(\frac{c_{A}^{\nu-1}}{c_{T}^{\nu_{t}}} \frac{\bar{z}}{z_{t}}\right)^{2} .
\end{aligned}
$$

The spectral indices are generally valid in all gravity theories we are considering in this work. In the generalized $f(\phi, R)$ gravity we have $r_{1}=4\left|\epsilon_{1}-\epsilon_{3}\right|=2\left|n_{T}\right|$. Thus, $r_{1}=4\left|\epsilon_{1}\right|=2\left|n_{T}\right|$ in the minimally coupled scalar field. The relation $r_{1}=2\left|n_{T}\right|$ in the minimally coupled scalar field is known as a 'consistency relation'. We notice that this relation is more generally valid in generalized $f(\phi, R)$ gravity. However, in the tachyonic correction we have

\footnotetext{
${ }^{4}$ In order to lift the square root we have assumed $3 \geq \epsilon_{1}-2 \epsilon_{2}+2 \epsilon_{3}-2 \epsilon_{4}$ and $3 \geq-\epsilon_{1}-2 \epsilon_{3}$.
} 
$r_{1}=4\left|\epsilon_{1}-\epsilon_{3}\right| c_{A}=2\left|n_{T}\right| c_{A}$, [19]; in a simpler case this result was presented in [35]. In the case of string correction terms we can derive

$$
r_{1}=4\left|\left\{\epsilon_{1}-\epsilon_{3}-\frac{1}{4 F}\left[\frac{1}{H^{2}}\left(2 Q_{c}+Q_{d}\right)-\frac{1}{H} Q_{e}+Q_{f}\right]\right\} \frac{1}{1+\frac{Q_{b}}{2 F}}\left(\frac{c_{A}}{c_{T}}\right)^{3}\right| .
$$

In the string axionic correction term we have

$$
r_{1}=4\left|\epsilon_{1}-\epsilon_{3}\right| \frac{1}{2} \sum_{\ell} \frac{1}{\left|1+2 \lambda_{\ell} \frac{k}{a} \frac{\dot{\nu}}{F}\right|} .
$$

Therefore, in the slow-roll limit $\epsilon_{i} \ll 1$ we have the tensor-type perturbation suppressed compared with the scalar-type perturbation. In the string corrections and the tachyonic correction we have non-trivial wave propagation speed $c_{A}$ (see Table 1; in the string correction case we have non-trivial $c_{T}$ as well, see Table 2) and the resulting scalar to tensor ratio $r_{1}$ could depend on the specific realization of the background evolution during the quantum generation stage. The result can be read from eq. (178) in the slow-roll limit, or eq. (161) in more general situation satisfying only eq. (133).

\section{DISCUSSIONS}

Considering our own publications on the subject, perhaps it would be useful to make clear the new points made in this work. Equations in terms of $\Phi$ and $\Psi$ for the generalized gravity theories in eqs. $(58,59,85,86,101,102)$, and the corresponding second-order equations in terms of $\Psi$ in eqs. $(62,90,105)$ are new. We have extended results in $\S$ III C and III E to the situation with general background curvature. Also, $\S I I I G$ is more general by considering general coupling of the field with gravity. We stress that the analyses in $\S I V$ and $\mathrm{V}$ are made in unified forms applicable to all the generalized gravity theories we have considered.

Notice that our $f(\phi, R)$ gravity includes $R^{2}$ gravity as a simple case. Although $R^{2}$-term in the action leads to a higher-order gravity theory, we have shown that we can derive second-order perturbation equations for the scalarand tensor-type perturbations in the context of $f(R)$ gravity. We have investigated the roles of $R^{a b} R_{a b}$ correction term separately in [36]; in four-dimensional spacetime, due to Gauss-Bonnet theorem, $R^{2}$ and $R^{a b} R_{a b}$ terms are complete fourth-order contributions by the pure curvature corrections to quadratic order. Contrary to $R^{2}$ gravity, the $R^{a b} R_{a b}$-term leads to fourth-order differential equations for both scalar- and tensor-type perturbations, see [36].

No rotational mode (vector-type perturbation) is directly excited by the presence of generalized forms of the scalarfield and the scalar-curvature; this is true even in the case of $R^{a b} R_{a b}$ gravity, [36]. This is because the evolution of rotational perturbation is simply described by the momentum-conservation equation of the additional fluid part energy-momentum tensor, $T_{(m) \alpha ; b} \stackrel{b}{b}=0$.

In this work we have considered only a single-component situation. In the multi-component situation our basic set of equations in eqs. (12-19) remains valid with the fluid quantities interpreted as the sum over the individual fluid quantities including fields. In order to describe the dynamics of the individual fluid or field component we additionally need the conservation equations of the individual energy-momentum tensor or the equation of motion. Such equations in Einstein's gravity limit and in the generalized $f(\phi, R)$ gravity are presented in [37] and [3,15], respectively. Extensions to more general situations with $S_{(c)}$ can be made similarly by considering the multiple fluids and fields in $T_{a b}^{(m)}$ in eqs. $(46,75)$.

We emphasize that most of our equations and analyses made in this work are independent of the specific scenarios of the universe, thus can be applied to the spatially homogenous and isotropic Friedmann world models based on our gravity theories. In a classic paper on the CMB anisotropies Sachs and Wolfe [38] has menthioned that

"the linear perturbations are so surprisingly simple that a perturbation analysis accurate to second order may be feasible..."

Considering our unified formulation of perturbations in such wide variety of gravity theories, the linear perturbations can perhaps be described as "surprisingly simple" indeed. Related to the second part of the statement, an accurate result in second order perturbation can be found in our recent work in [39]. 


\section{ACKNOWLEDGMENTS}

HN was supported by grant No. R04-2003-10004-0 from the Basic Research Program of the Korea Science and Engineering Foundation. JH was supported by the Korea Research Foundation Grants 2003-015-C00253.

[1] E.M. Lifshitz, J. Phys. (USSR) 10, 116 (1946); E.M. Lifshitz and I.M. Khalatnikov, Adv. Phys. 12, 185 (1963).

[2] J.M. Bardeen, in Cosmology and Particle Physics, edited by L. Fang and A. Zee (Gordon and Breach, London, 1988), p. 1.

[3] J. Hwang, Astrophys. J. 375, 443 (1991).

[4] J. Ehlers, Proceedings of the mathematical-natural science of the Mainz academy of science and literature, Nr. 11, 792 (1961), translated in Gen. Rel. Grav. 25, 1225 (1993); G.F.R. Ellis, in General relativity and cosmology, Proceedings of the international summer school of physics Enrico Fermi course 47, edited by R. K. Sachs (Academic Press, New York, 1971), 104; in Cargese Lectures in Physics, edited by E. Schatzmann (Gorden and Breach, New York, 1973 ), 1.

[5] J.M. Bardeen, Phys. Rev. D 22, 1882 (1980).

[6] J. Hwang, Class. Quant. Grav. 7, 1613 (1990).

[7] G.B. Field and L.C. Shepley, Astrophys. Space. Sci. 1, 309 (1968).

[8] J. Hwang, Phys. Rev. D 60, 103512 (1999).

[9] J. Hwang and H. Noh, Gen. Rel. Grav. 31, 1131 (1999).

[10] E.R. Harrison, Rev. Mod. Phys. 39, 862 (1967).

[11] H. Nariai, Prog. Theor. Phys. 41, 686 (1969).

[12] V.F. Mukhanov, Sov. Phys. JETP Lett. 41, 493 (1985).

[13] V.F. Mukhanov, Sov. Phys. JETP 68, 1297 (1988).

[14] J. Hwang, Phys. Rev. D 48, 3544 (1993); Class. Quant. Grav. 11, 2305 (1994); Astrophys. J. 427, 542 (1994).

[15] J. Hwang and H. Noh, Phys. Rev. D 65, 023512 (2002).

[16] J. Hwang, Phys. Rev. D 53, 762 (1996).

[17] J. Hwang and H. Noh, Phys. Rev. D 54, 1460 (1996).

[18] J. Hwang, Class. Quant. Grav. 14, 1981 (1997).

[19] J. Hwang and H. Noh, Phys. Rev. D 66, 084009 (2002).

[20] J. Hwang and H. Noh, Phys. Rev. D 61, 043511 (2000).

[21] C. Cartier, J. Hwang and E. Copeland, Phys. Rev. D 64103504 (2001).

[22] K. Choi, J. Hwang and K.W. Hwang, Phys. Rev. D 61, 084026 (2000).

[23] L.H. Ford and L. Parker, Phys. Rev. D 16, 1601 (1977).

[24] J. Hwang, Class. Quant. Grav. 14, 3327 (1997).

[25] N.D. Birrell and P.C.W. Davies, Quantum fields in curved space (Cambridge Univ. Press, Cambridge, 1982).

[26] J. Hwang, Class. Quant. Grav. 15, 1401 (1998).

[27] J. Hwang and H. Noh, Phys. Rev. D 65, 124010 (2002).

[28] V.N. Lukash, Sov. Phys. JETP Lett. 31, 596 (1980); Sov. Phys. JETP 52, 807 (1980); G.V. Chibisov and V.F. Mukhanov, Mon. Not. R. Astron. Soc. 200, 535 (1982); V.F. Mukhanov, H.A. Feldman and R.H. Brandenberger, Phys. Rep. 215, 203 (1992).

[29] A.H. Guth and S. Pi, Phys. Rev. Lett. 49, 1110 (1982).

[30] L. Knox, Phys. Rev. D 52, 4307 (1995).

[31] C.L. Bennett, et al, ApJ. 464, L1 (1996).

[32] C.L. Bennett, et al, Astrophys. J. Suppl. 148, 175 (2003).

[33] E.D. Stewart and D.H. Lyth, Phys. Lett. B 302, 171 (1993).

[34] E.D. Stewart and J. Gong, Phys. Lett. B 510, 1 (2001); S. Habib, K. Heitmann, G. Jungman and C. Molina-Paris, Phys. Rev. Lett. 89, 281301 (2002); E.D. Stewart, Phys. Rev. D 65, 103508 (2002); J. Martin and D. J. Schwarz, Phys. Rev. D 67, 083512 (2003); J. Choe, J. Gong and E.D. Stewart, hep-ph/0405155; S. Habib, A. Heinen, K. Heitmann, G. Jungman and C. Molina-Paris, astro-ph/0406134.

[35] J. Garriga and V. F. Mukhanov, Phys. Lett. B 458, 219 (1999).

[36] H. Noh and J. Hwang, Phys. Rev. D 55, 5222 (1997); 57, 2617 (1998); 59, 047501 (1999).

[37] J. Hwang and H. Noh, Class. Quant. Grav. 19, 527 (2002).

[38] R.K. Sachs and A.M. Wolfe, Astrophys. J. 147, 73 (1967).

[39] H. Noh and J. Hwang, Phys. Rev. D 69, 104011 (2004); see next papers posted in astro-ph. 
Table 1. Scalar-type perturbation: We present the coefficients and definitions used in our unified formulations of the scalar-type perturbation in $\S \mathrm{IV}$ and V. We introduce $x_{4} \equiv \omega \dot{\phi}^{2}+3 \frac{\left(\dot{F}+Q_{a}\right)^{2}}{2 F+Q_{b}}+Q_{c}+Q_{d}+\frac{\dot{F}+Q_{a}}{2 F+Q_{b}} Q_{e}+\left(\frac{\dot{F}+Q_{a}}{2 F+Q_{b}}\right)^{2} Q_{f}$. Except for the string corrections in the last column, the other situations are valid considering general $K$; for $c_{A}^{2}$ we present result assuming $K=0$.

\begin{tabular}{|c|c|c|c|c|c|}
\hline & Fluid & Field & $f(\phi, R)$ gravity & Tachyonic & String corrections \\
\hline$\Phi \equiv$ & $\varphi_{v}-\frac{K}{a^{2}} \frac{1}{4 \pi G(\mu+p)} \varphi_{\chi}$ & $\varphi_{v}-\frac{K}{a^{2}} \frac{1}{4 \pi G \dot{\phi}^{2}} \varphi_{\chi}$ & $\varphi_{\delta \phi}-\frac{K}{a^{2}} \frac{2 F}{\omega \dot{\phi}^{2}+\frac{3 \dot{F}^{2}}{2 F}} \Psi$ & $\varphi_{\delta \phi}-\frac{K}{a^{2}} \frac{2 F}{X f, X+\frac{3 F^{2}}{2 F}} \Psi$ & $\varphi_{\delta \phi}$ \\
\hline$\Psi \equiv$ & $\varphi_{\chi}$ & $\varphi_{\chi}$ & $\varphi_{\chi}+\frac{\delta F_{\chi}}{2 F}$ & $\varphi_{\chi}+\frac{\delta F_{\chi}}{2 F}$ & $\varphi_{\chi}+\frac{\dot{F}+Q_{a}}{2 F+Q_{b}} \frac{\delta F_{\chi}}{\dot{F}}$ \\
\hline$x_{1} \equiv$ & $\frac{H}{8 \pi G(\mu+p)} c_{s}^{2}$ & $\frac{H}{8 \pi G \dot{\phi}^{2}} c_{A}^{2}$ & $\frac{H F+\frac{1}{2} \dot{F}}{\omega \dot{\phi}^{2}+\frac{3 F^{2}}{2 F}} c_{A}^{2}$ & $\frac{H F+\frac{1}{2} \dot{F}}{X f, X+\frac{3 F^{2}}{2 F}} c_{A}^{2}$ & $\frac{\left(H+\frac{\dot{F}+Q_{a}}{2 F+Q_{b}}\right)\left(F+\frac{1}{2} Q_{b}\right)}{\omega \dot{\phi}^{2}+3 \frac{\left(\dot{F}+Q_{a}\right)^{2}}{2 F+Q_{b}}+Q_{c}}$ \\
\hline$x_{2} \equiv$ & $\frac{1}{8 \pi G} \frac{a}{H}$ & $\frac{1}{8 \pi G} \frac{a}{H}$ & $\frac{a F}{H+\frac{\vec{F}}{2 F}}$ & $\frac{a F}{H+\frac{F}{2 F}}$ & $\frac{a\left(F+\frac{1}{2} Q_{b}\right)}{H+\frac{\dot{F}+Q_{a}}{2 F+Q_{b}}}$ \\
\hline$x_{3} \equiv$ & $8 \pi G \frac{\mu+p}{H}$ & $8 \pi G \frac{\dot{\phi}^{2}}{H}$ & $\frac{\omega \dot{\phi}^{2}+\frac{3 \dot{F}^{2}}{2 F}}{H F+\frac{1}{2} \dot{F}}$ & $\frac{X f, X+\frac{3 \dot{F}^{2}}{2 F}}{H F+\frac{1}{2} \dot{F}}$ & $\frac{1}{\left(H+\frac{\dot{F}+Q_{a}}{2 F+Q_{b}}\right)\left(F+\frac{1}{2} Q_{b}\right)} x_{4}$ \\
\hline$c_{A}^{2} \equiv$ & $c_{s}^{2}\left(\equiv \frac{\dot{p}}{\dot{\mu}}\right)$ & 1 & 1 & $\frac{X f, X+\frac{3 \dot{F}^{2}}{2 F}}{X f, X+2 X^{2} f, X X+\frac{3 \dot{F}^{2}}{2 F}}$ & $\frac{x_{4}}{\omega \dot{\phi}^{2}+3 \frac{\left(\dot{F}+Q_{a}\right)^{2}}{2 F+Q_{b}}+Q_{c}}$ \\
\hline $\bar{z} \equiv$ & $\frac{a}{H} \sqrt{\mu+p}$ & $\frac{a}{H} \dot{\phi}$ & $\frac{a}{H+\frac{F}{2 F}} \sqrt{\omega \dot{\phi}^{2}+\frac{3 \dot{F}^{2}}{2 F}}$ & $\frac{a}{H+\frac{F}{2 F}} \sqrt{X f_{, X}+\frac{3 \dot{F}^{2}}{2 F}}$ & $\frac{a}{H+\frac{F+Q_{a}}{2 F+Q_{b}}} \sqrt{x_{4}}$ \\
\hline$u \equiv$ & $\frac{1}{8 \pi G \sqrt{\mu+p}} \Psi$ & $\frac{1}{8 \pi G \phi} \Psi$ & $\frac{F}{\sqrt{\omega \dot{\phi}^{2}+\frac{3 \dot{F}^{2}}{2 F}}} \Psi$ & $\frac{F}{\sqrt{X f, X+\frac{3 \dot{F}^{2}}{2 F}}} \Psi$ & $\frac{F+\frac{1}{2} Q_{b}}{\sqrt{x_{4}}} \Psi$ \\
\hline
\end{tabular}

Table 2. Tensor-type perturbation: Continuation of Table 1 for the tensor-type perturbation (gravitational wave). In the cases of the string corrections and the string axion we assume $K=0$; for $c_{T}^{2}$ we present result assuming $K=0$.

\begin{tabular}{|c|c|c|c|c|}
\hline & Fluid, Field & $f(\phi, R)$ gravity, Tachyonic & String corrections & String axion \\
\hline$z_{t} \equiv$ & $a \frac{1}{\sqrt{8 \pi G}}$ & $a \sqrt{F}$ & $a \sqrt{F+\frac{1}{2} Q_{b}}$ & $a \sqrt{F+2 \lambda_{\ell} \dot{\nu} k / a}$ \\
\hline$c_{T}^{2} \equiv$ & 1 & 1 & $1+\frac{Q_{f}}{2 F+Q_{b}}$ & 1 \\
\hline
\end{tabular}

